\title{
Wave-equation $\mathbf{Q}_{s}$ Inversion of Skeletonized Surface Waves
}

\author{
Jing $\mathrm{Li}^{1}$, Gaurav Dutta ${ }^{1}$ and Gerard Schuster ${ }^{1}$ \\ ${ }^{1}$ King Abdullah University of Science and Technology (KAUST), Thuwal 23955-6900, Kingdom of Saudi Arabia. \\ Email: inter.lijing@gmail.com
}

8 February 2017

\section{SUMMARY}

We present a skeletonized inversion method that inverts surface-wave data for the $\mathrm{Q}_{s}$ quality factor. Similar to the inversion of dispersion curves for the S-wave velocity model, the complicated surface-wave arrivals are skeletonized as simpler data, namely the amplitude spectra of the windowed Rayleigh-wave arrivals. The optimal $Q_{s}$ model is the one that minimizes the difference in the peak frequencies of the predicted and observed Rayleigh wave arrivals using a gradient-based wave-equation optimization method. Solutions to the viscoelastic waveequation are used to compute the predicted Rayleigh-wave arrivals and the misfit gradient at every iteration. This procedure, denoted as wave-equation $\mathrm{Q}_{s}$ inversion $\left(\mathrm{WQ}_{s}\right)$, does not require the assumption of a layered model and tends to have fast and robust convergence compared to full waveform inversion (FWI). Numerical examples with synthetic and field data demonstrate that the $\mathrm{WQ}_{s}$ method can accurately invert for a smoothed approximation to the subsurface $\mathrm{Q}_{s}$ distribution as long as the $\mathrm{V}_{s}$ model is known with sufficient accuracy.

Key words: Surface waves, attenuation, Skeletonized $\mathrm{Q}_{s}$ inversion.

\section{INTRODUCTION}

Surface waves play an important role in the characterization of the near surface for earthquake, engineering and environmental studies. Inverting and imaging surface waves can be an effective means for characterizing the subsurface at different scales (Dasgupta \& Clark 1998; 


\section{Jing Li, et al.}

Xia et al. 1999; Lin et al. 2008; Boiero et al. 2013; Li \& Hanafy 2016), and can be important for site response and seismic hazard studies. Since the surface waves are sensitive to the near-surface elastic properties, estimation of the surface-wave velocity $\mathrm{V}_{s}$ and quality factor $\mathrm{Q}_{s}$ are of significant interest in exploration and earthquake seismology (Strobbia et al. 2011).

The near-surface S-wave velocity model is usually estimated from the dispersion curves of the recorded surface-wave arrivals (Park et al. 1998). However, in a real dissipative media, the propagation of the surface waves is strongly influenced by elastic damping in the near-surface that results in increasing amplitude loss and attenuation of high frequencies with distance travelled. As a result, the dispersion curves are also sensitive to the near-surface $\mathrm{Q}_{s}$ variations. He et al. (2015) showed that the phase velocity of the fundamental mode of the Rayleigh waves increases with decreasing values of $\mathrm{Q}_{s}$ and the phase velocity increases with increasing values of the Poison s ratio. Thus, inverting dispersion curves for the $\mathrm{S}$-wave velocity without taking into consideration the effect of $\mathrm{Q}_{s}$ can lead to erroneous estimates of the near-surface S-velocity distribution. Groos et al. (2014) showed that the S-wave velocity tomograms obtained from elastic full waveform inversion (FWI) of Rayleigh waves have lower resolution when compared to the tomograms obtained by viscoelastic FWI when the nearsurface is strongly anelastic. The increased resolution in S-wave tomograms obtained by taking into account the effect of $\mathrm{Q}_{s}$ can be useful in delineating near-surface faults or local anomalies (Pinson et al. 2008).

The conventional method for $\mathrm{Q}_{s}$ estimation is based on the linear relationship between the Rayleigh-wave attenuation coefficients, the quality factors $\mathrm{Q}_{p}$ and $\mathrm{Q}_{s}$ and the $\mathrm{P}$ - and $\mathrm{S}$-wave velocities (Anderson et al. 1965). This relationship is valid for a layered medium and is given by

$$
\alpha_{R}(f)=\frac{\pi f}{C_{R}^{2}(f)}\left[\sum_{i=1}^{n} P_{i}(f) Q_{p i}^{-1}+\sum_{i=1}^{n} S_{i}(f) Q_{s i}^{-1}\right]
$$

where

$$
P_{i}(f)=V_{p i} \frac{\partial C_{R}(f)}{\partial V_{p i}}, \text { and } S_{i}(f)=V_{s i} \frac{\partial C_{R}(f)}{\partial V_{s i}}
$$

Here $\alpha_{R}(f)$ is the Rayleigh waves attenuation coefficient for the frequency $f, \mathrm{~V}_{p i}$ and $\mathrm{V}_{s i}$ are the P-and S-wave velocities, respectively, in the ith layer, $C_{R}$ is the Rayleigh-wave phase-velocity and $n$ is the number of layers in the model. Xia et al. ( 2002), Xia (2014) and Ivanov et al. (2014) proposed different methods for estimating $\mathrm{Q}_{p}$ and $\mathrm{Q}_{s}$ based on this empirical relationship. Boiero et al. (2013) used a least-squares type approach and equation 1 to iteratively update the values of both $\mathrm{Q}_{p}$ and $\mathrm{Q}_{s}$. However, all these approaches are based on a layered-medium assumption and do no take into account strong lateral gradients in $\mathrm{V}_{s}$ model.

Other approaches for estimating $\mathrm{Q}_{s}$ are based on the spectral-ratio method where the logarithmic amplitude ratios are plotted against the arrival-time difference recorded at any two receivers for a specified frequency. The slope of the regression line then gives an estimate of the $\mathrm{Q}_{s}$ model for each frequency (Jeng et al. 1999; Li et al. 2016). An inverse Q-filtering type approach can then be used to compensate for the amplitude loss in the data-domain. Parolai et al. (2010) estimated the average $\mathrm{Q}_{s}$ down to any depth level by using surface seismic 
data to deconvolve the wavefield recorded in boreholes by vertical-array accelerometers. Their approach is based on the assumption that the impedance contrasts in the layers between the surface and the investigated depth range are small and the deconvolved wavefield is dominated by vertically propagating S-waves.

In viscoacoustic media, a $\mathrm{Q}_{p}$ model can be estimated using wave-equation tomographic techniques. As an example, Quan and Harris (1997) computed the centroid-frequency shifts between the predicted and observed traces, and then smeared the shifts along raypaths to update the Q model. This approach used ray tracing so it assumes a high-frequency approximation of the data. Alternative approaches include using full-waveform inversion (FWI), in which the Q model is found that minimizes the difference between the observed and calculated traces (Bai \& Yingst 2013; Bai et al. 2014; Wang \& Zhang 2014). Similar to other FWI methods, this approach is prone to getting stuck in local minima.

To avoid the layered medium assumption as well the local minimum problems associated with FWI of surface-wave traces, we present a novel wave-equation method that inverts skeletonized surface waves for the quality factor $\mathrm{Q}_{s}$. In our method denoted as $\mathrm{WQ}_{s}$, the skeletonized data are the frequency shifts of the surface-wave's spectral peaks as they propagate to greater distances. The $Q_{s}$ method is similar to the waveequation $Q_{p}$ inversion developed by Dutta and Schuster (2016), except it inverts for $Q_{s}$ from surface waves rather than $Q_{p}$ from body-wave arrivals. Here, the observed and predicted Rayleigh-wave arrivals are skeletonized to a simple representation in the form of peak frequencies in their amplitude spectra. The $\mathrm{Q}_{s}$ model is found that minimizes the squared differences between the predicted and observed peak-frequency shifts associated with the Rayleigh wave arrivals. For this method, the isotropic viscoelastic wave equation based on the standard linear solid model (Robertsson et al. 1994) is used to generate the predicted Rayleigh-wave arrivals. The adjoint viscoelastic wave-equation is then used to backpropagate the residual traces that are obtained by weighting the observed Rayleigh-wave arrivals with their corresponding frequency shifts. The gradient $\gamma(\mathbf{x})$ for $\mathrm{WQ}_{s}$ is derived using the implicit function theorem (Luo \& Schuster 1991a; Luo \& Schuster 1991b) and the adjoint-state method (Plessix 2006), and $\gamma(\mathbf{x})$ can be interpreted as the zero-lag cross-correlation between the forward propagated source wavefield and the weighted backprojected residual wavefield. The proposed approach has no layered-medium assumption and can handle complex near-surface heterogeneities. The near-surface $\mathrm{Q}_{s}$ distribution can be inverted by using recordings only at the surface and there is no requirement for having receivers at different depth levels, which is often a necessity for deconvolution-based $\mathrm{Q}_{s}$ estimation approaches (Xia et al. 2002).

This paper is organized into four sections. After the introduction, the second section describes the theory of skeletonized surface-wave $\mathrm{Q}_{s}$ inversion. Numerical results for synthetic and field data from a 2D near-surface survey conducted in the Qademah region of Saudi Arabia are then presented in the third section. The discussions and conclusions are in the last section. 


\section{THEORY OF WAVE-EQUATION $Q_{S}$ SKELETONIZED INVERSION}

The theory of $\mathrm{WQ}_{s}$ is derived in a manner that is similar to that for wave-equation traveltime inversion (WT) (Luo \& Schuster 1991a) and surface-wave dispersion inversion (WD) (Li et al. 2017). These steps include: (1) define a frequency-shift misfit function, (2) define a connective function (Luo \& Schuster 1991b) that connects the frequency-shift residual of the Rayleigh-wave arrivals in the observed and predicted seismogram spectra, and (3) derive the gradient of the misfit function with respect to $\mathrm{Q}_{s}$ using the isotropic viscoelastic wave equation and the connective function.

In our analysis, we assume that the wave propagation honors the $2 \mathrm{D}$ isotropic viscoelastic equations of motion based on the standard linear solid (SLS) mechanism (Blanch et al.1995)

$$
\begin{aligned}
\rho \frac{\partial u}{\partial t} & =\frac{\partial \sigma_{x x}}{\partial x}+\frac{\partial \sigma_{x z}}{\partial z} \\
\rho \frac{\partial w}{\partial t} & =\frac{\partial \sigma_{z x}}{\partial x}+\frac{\partial \sigma_{z z}}{\partial z}, \\
\frac{\partial \sigma_{x x}}{\partial t} & =\pi \frac{\tau_{\epsilon}^{p}}{\tau_{\sigma}}\left(\frac{\partial u}{\partial x}+\frac{\partial w}{\partial z}\right)-2 \mu \frac{\tau_{\epsilon}^{s}}{\tau_{\sigma}} \frac{\partial w}{\partial z}+r_{x x}+S_{x x}, \\
\frac{\partial \sigma_{z z}}{\partial t} & =\pi \frac{\tau_{\epsilon}^{p}}{\tau_{\sigma}}\left(\frac{\partial u}{\partial x}+\frac{\partial w}{\partial z}\right)-2 \mu \frac{\tau_{\epsilon}^{s}}{\tau_{\sigma}} \frac{\partial u}{\partial x}+r_{z z}+S_{z z}, \\
\frac{\partial \sigma_{x z}}{\partial t} & =\mu \frac{\tau_{\epsilon}^{s}}{\tau_{\sigma}}\left(\frac{\partial w}{\partial x}+\frac{\partial u}{\partial z}\right)+r_{x z}, \\
\frac{\partial r_{x x}}{\partial t} & =-\frac{1}{\tau_{\sigma}}\left(r_{x x}+\pi\left(\frac{\tau_{\epsilon}^{p}}{\tau_{\sigma}}-1\right)\left(\frac{\partial u}{\partial x}+\frac{\partial w}{\partial z}\right)-2 \mu\left(\frac{\tau_{\epsilon}^{s}}{\tau_{\sigma}}-1\right) \frac{\partial w}{\partial z}\right), \\
\frac{\partial r_{z z}}{\partial t} & =-\frac{1}{\tau_{\sigma}}\left(r_{z z}+\pi\left(\frac{\tau_{\epsilon}^{p}}{\tau_{\sigma}}-1\right)\left(\frac{\partial u}{\partial x}+\frac{\partial w}{\partial z}\right)-2 \mu\left(\frac{\tau_{\epsilon}^{s}}{\tau_{\sigma}}-1\right) \frac{\partial u}{\partial x}\right), \\
\frac{\partial r_{x z}}{\partial t} & =-\frac{1}{\tau_{\sigma}}\left(r_{x z}+\mu\left(\frac{\tau_{\epsilon}^{s}}{\tau_{\sigma}}-1\right)\left(\frac{\partial u}{\partial z}+\frac{\partial w}{\partial x}\right)\right) .
\end{aligned}
$$

Here, $u$ and $w$ are the horizontal- and vertical-particle-velocity components, $\sigma_{i j}$ denotes the $i j$-th component of the symmetric stress tensor, $r_{i j}$ is the memory variable, $\tau_{\epsilon}^{p}$ and $\tau_{\epsilon}^{s}$ are the strain relaxation times for the $\mathrm{P}$ and $\mathrm{SV}$ waves, and $\tau_{\sigma}$ is the stress relaxation time for both the P and SV waves. $S_{x x}$ and $S_{z z}$ denote the source wavelets for the special case of an explosive source. The variable $\pi=\lambda+2 \mu$ is related to the Lamé parameters $\lambda$ and $\mu$ whereas the stress and strain relaxation times are related to the quality factors $\mathrm{Q}_{p}$ and $\mathrm{Q}_{s}$ as $\left(\mathrm{Carcione}_{2001)}\right.$

$$
\begin{aligned}
\tau_{\sigma} & =\frac{\sqrt{1+\frac{1}{Q_{p}^{2}}}-\frac{1}{Q_{p}}}{\omega}, \\
\tau_{\epsilon}^{s} & =\frac{1+\omega Q_{s} \tau_{\sigma}}{\omega Q_{s}-\omega^{2} \tau_{\sigma}}, \\
\tau_{\epsilon}^{p} & =\frac{1}{\omega^{2} \tau_{\sigma}} .
\end{aligned}
$$

Here, the stress and strain relaxation parameters, $\tau_{\epsilon}^{p}$ and $\tau_{\epsilon}^{s}$, are related to the quality factor $\mathbf{Q}_{s}$, and the reference angular frequency is $\omega$ (Robertsson et al. 1994). Equation 3 is solved for a point source at each shot point by an $\mathrm{O}(2,4)$ time-space staggered grid finite-difference algorithm. In order to generate surface waves, an explicit free-surface boundary condition is implemented by using the mirroring technique 
proposed by Levander (1988). Blanch et al. (1995) suggested the use of the $\tau$-method to reduce the number of SLS variables and therefore, reduce the calculation time and the memory requirements.

It is possible to approximate a frequency independent seismic quality factor in a limited frequency range. We define the frequencyindependent parameter $\eta$ :

$$
\eta=\frac{\tau_{\epsilon}{ }^{s}}{\tau_{\sigma}}-1=\frac{1+\left(\sqrt{1+\frac{1}{Q_{p}^{2}}}-\frac{1}{Q_{p}}\right)^{2}}{\left(\sqrt{1+\frac{1}{Q_{p}^{2}}}-\frac{1}{Q_{p}}\right)\left[Q_{s}-\left(\sqrt{1+\frac{1}{Q_{p}^{2}}}-\frac{1}{Q_{p}}\right)\right]} .
$$

For realistic geologic models, the $\mathrm{Q}_{p}$ varies from 20 to 200 , so that $\sqrt{1+\frac{1}{Q_{p}^{2}}}-\frac{1}{Q_{p}}$ is almost equal to 1 . Thus, for the parametrization in $\mathrm{WQ}_{s}, \eta$ is used because it is quite sensitive to small changes in $\mathrm{Q}_{s}$. The relaxation ratio $\eta$ is inverted at each iteration and the updates in $\eta$ are then mapped to $\mathrm{Q}_{s}$ using the relations given in equation 4 .

\subsection{Misfit Function}

We denote the Rayleigh-wave arrivals that are extracted from the recorded data as $D_{f_{g}^{o b s}}\left(\mathbf{x}_{g}, t ; \mathbf{x}_{s}\right)^{o b s}$ for a vertical-component point source at $\mathbf{x}_{s}$ and a vertical-component receiver at $\mathbf{x}_{g}$ (the black curve in Figure 1a). The predicted Rayleigh-wave arrivals for the same sourcereceiver pair are denoted by $D_{f_{g}^{p r e d}}\left(\mathbf{x}_{g}, t ; \mathbf{x}_{s}\right)$ (the red curve in Figure 1a). The peak frequency of the observed and predicted spectra are denoted as $f_{g}^{o b s}$ and $f_{g}^{\text {pred }}$, respectively. Here, $f_{g}^{\text {pred }}=f$ and $f_{g}^{o b s}=f-f_{1}$, where $f$ is the peak frequency of the event and $f_{1}$ is the shift between the peak frequencies of the predicted and the observed traces. A comparison between the windowed observed and predicted Rayleigh-wave arrivals for a given $\mathrm{Q}_{s}$ model is shown in Figure 1b. The amplitude spectra of these arrivals are plotted in Figure 1c, where it is evident that the observed spectrum has a lower peak frequency than the predicted spectrum. The peak frequencies in these spectra are denoted as the skeletonized surface-wave data.

The goal of $\mathrm{WQ}_{s}$ is to find the attenuation model $\mathrm{Q}_{s}=F(\eta(\mathbf{x}))^{-1}$ so that $f_{g}^{\text {pred }} \approx f_{g}^{o b s}$ for all the traces. In our case, we use the frequency-shift residual $\Delta f=f_{g}^{\text {pred }}-f_{g}^{o b s}$ to form the skeletonized misfit function:

$$
\epsilon=\frac{1}{2} \sum_{g} \sum_{s} \Delta f\left(\mathbf{x}_{g}, \mathbf{x}_{s}\right)^{2}
$$

The gradient $\gamma(\mathbf{x})$ is given by

$$
\gamma(\mathbf{x})=\frac{\partial \epsilon}{\partial \eta(\mathbf{x})}=\sum_{g} \sum_{s} \frac{\partial \Delta f}{\partial \eta(\mathbf{x})} \Delta f\left(\mathbf{x}_{g}, \mathbf{x}_{s}\right)
$$



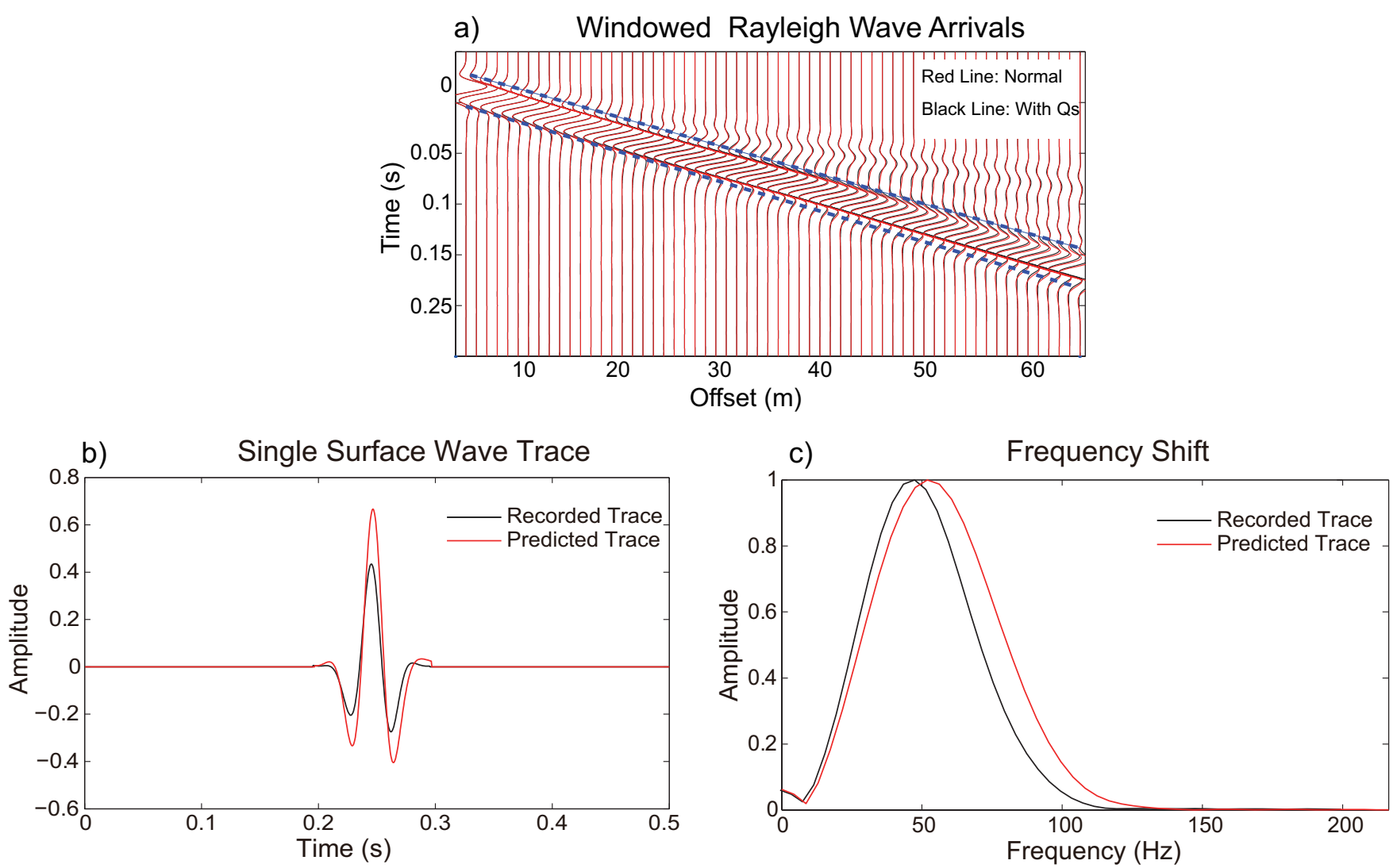

Figure 1. (a) A common shot gather (CSG) comparing the Rayleigh-wave arrivals with and without $\mathrm{Q}_{s}$. The blue dashed line shows the window used to extract these arrivals for $\mathrm{WQ}_{s}$, (b) comparison between a predicted and an observed surface-wave arrival, and (c) comparison between their frequency spectra.

\subsection{Connective Function}

To find an analytic expression for the Fréchet derivative $\frac{\partial \Delta f}{\partial \eta(\mathbf{x})}$ in equation 7 , we define the connective function $\Phi\left(\mathbf{x}_{g}, t ; \mathbf{x}_{s}\right)$ that connects the change in peak frequency of an arrival with the observed and the predicted Rayleigh-wave arrivals in Figure 1b as (Dutta \& Schuster 2016)

$$
\Phi_{f_{1}}\left(\mathbf{x}_{g}, t ; \mathbf{x}_{s}\right)=\int D_{f}\left(\mathbf{x}_{g}, t ; \mathbf{x}_{s}\right) D_{f-f_{1}}\left(\mathbf{x}_{g}, t ; \mathbf{x}_{s}\right)^{o b s} d t
$$

We seek the optimal $\mathrm{Q}_{s}$ model that minimizes the peak-frequency shift between an observed and a predicted traces. For an accurate background $\mathrm{Q}_{s}$ model, the predicted and the observed arrivals will have the same peak frequency. So, we define $f_{1}=\Delta f$ to be the frequency shift associated with the actual background $\mathrm{Q}_{s}$ model. If $\Delta f=0$, it indicates that the correct background $\mathrm{Q}_{s}$ model has been found and the transmission surface-wave arrivals in the predicted and observed traces have the same peak frequencies. The derivative of $\Phi_{f_{1}}$ with respect to $f_{1}$ should be zero at the frequency-shift value $f_{1}=\Delta f$, i.e.,

$$
\dot{\Phi}_{\Delta f}(\Delta f, \eta(\mathbf{x}))=\left[\frac{\partial \Phi_{f_{1}}\left(\mathbf{x}_{g}, t ; \mathbf{x}_{s}\right)}{\partial f_{1}}\right]_{f_{1}=\Delta f}=\int D_{f}\left(\mathbf{x}_{g}, t ; \mathbf{x}_{s}\right) \dot{D}_{f-\Delta f}\left(\mathbf{x}_{g}, t ; \mathbf{x}_{s}\right)^{o b s} d t=0
$$

where $\dot{D}_{f-\Delta f}\left(\mathbf{x}_{g}, t ; \mathbf{x}_{s}\right)^{o b s}=\left[\partial D_{f-f_{1}}\left(\mathbf{x}_{g}, t ; \mathbf{x}_{s}\right)^{o b s} / \partial f_{1}\right]_{f_{1}=\Delta f}$. Note that $\Delta f=0$ if the predicted $\mathrm{Q}_{s}$ model is the actual $\mathrm{Q}_{s}$ model. Equation 9 is the connective function (Luo \& Schuster 1991a; Luo \& Schuster 1991b) that connects the skeletonized data, i.e., the frequency- 
shift residuals of the Rayleigh-wave arrivals, with the particle-velocity seismograms. Such a connective function is required because there is no wave equation that relates the skeletonized data to a single type of model parameter (Dutta \& Schuster 2016).

Using the implicit function theorem and the connective function in equation 9, the Fréchet derivative with respect to the relaxation ratio $\eta(\mathbf{x})$ can be expressed as

$$
\frac{\partial \Delta f}{\partial \eta(\mathbf{x})}=-\frac{\partial \dot{\Phi}}{\partial \eta(\mathbf{x})} / \frac{\partial \dot{\Phi}}{\partial \Delta f}
$$

where the numerator on the right-hand side is given by

$$
\frac{\partial \dot{\Phi}_{\Delta f}}{\partial \eta(\mathbf{x})}=\int \frac{\partial D_{f}\left(\mathbf{x}_{g}, t ; \mathbf{x}_{s}\right)}{\partial \eta(\mathbf{x})} \dot{D}_{f-\Delta f}\left(\mathbf{x}_{g}, t ; \mathbf{x}_{s}\right)^{o b s} d t
$$

and the denominator by

$$
\frac{\partial \dot{\Phi}_{\Delta f}}{\partial \Delta f}=\int D_{f}\left(\mathbf{x}_{g}, t ; \mathbf{x}_{s}\right) \ddot{D}_{f-\Delta f}\left(\mathbf{x}_{g}, t ; \mathbf{x}_{s}\right)^{o b s} d t=K_{2}
$$

Using equation 10 , the gradient in equation 7 can be written as

$$
\gamma(\mathbf{x})=\frac{\partial \epsilon}{\partial \eta(\mathbf{x})}=-\sum_{g} \sum_{s} \frac{\frac{\partial \dot{\Phi}}{\partial \eta(\mathbf{x})}}{\frac{\partial \dot{\Phi}}{\partial \Delta f}} \Delta f\left(\mathbf{x}_{g}, \mathbf{x}_{s}\right) .
$$

Then, we use the adjoint-state method to derive the Fréchet derivative shown in Appendix A. Combining equations 11-13, the gradient for $\mathrm{WQ}_{s}$ can be written as

$$
\begin{aligned}
\gamma(\mathbf{x}) & =-\sum_{s} \sum_{g} \frac{1}{K_{2}} \frac{\partial \dot{\Phi}}{\partial \eta(\mathbf{x})} \Delta f \\
& =-\sum_{s} \int\left(2 \mu \frac{\partial w}{\partial z} \hat{\sigma}_{x x}+2 \mu \frac{\partial u}{\partial x} \hat{\sigma}_{z z}-\left(\mu \frac{\partial u}{\partial z}+\mu \frac{\partial w}{\partial x}\right) \hat{\sigma}_{x z}-2 \mu E \frac{\partial w}{\partial z} \hat{r}_{x x}-2 \mu E \frac{\partial u}{\partial x} \hat{r}_{z z}+\mu E\left(\frac{\partial w}{\partial x}+\frac{\partial u}{\partial z}\right) \hat{r}_{x z}\right) d t
\end{aligned}
$$

Here $\left(\hat{u}, \hat{w}, \hat{\sigma}_{x x}, \hat{\sigma}_{z z}, \hat{\sigma}_{x z}, \hat{r}_{x x}, \hat{r}_{z z}, \hat{r}_{x z}\right)$ are the adjoint-state variables of $\left(u, w, \sigma_{x x}, \sigma_{z z}, \sigma_{x z}, r_{x x}, r_{z z}, r_{x z}\right)$ derived in Appendix A and $\mathrm{E}$ is defined in equation A.3. The gradient in equation 14 can be interpreted as a zero-lag crosscorrelation between the source-wavefield terms $u, w$ and the backpropagated residual wavefields $\hat{\sigma}_{x x}, \hat{\sigma}_{z z}, \hat{\sigma}_{x z}, \hat{r}_{x x}, \hat{r}_{z z}$ and $\hat{r}_{x z}$. The residual wavefields are computed by backpropagating the residual traces using the adjoint viscoelastic wave equation in equation A.9. The residual traces are obtained by weighting the observed Rayleigh-wave arrivals with their corresponding frequency shifts.

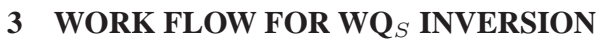

The following steps describe the workflow for numerically implementing $\mathrm{WQ}_{s}$ using a steepest descent method (Nocedal \& Wright 1999):

(i) Window the Rayleigh-wave arrivals in the data.

(ii) Apply a Fourier transform to the observed and the predicted Rayleigh-wave arrivals to get their amplitude spectra.

(iii) From the amplitude spectra of the traces, estimate the peak-frequencies or the centroid-frequencies and form the misfit function. 

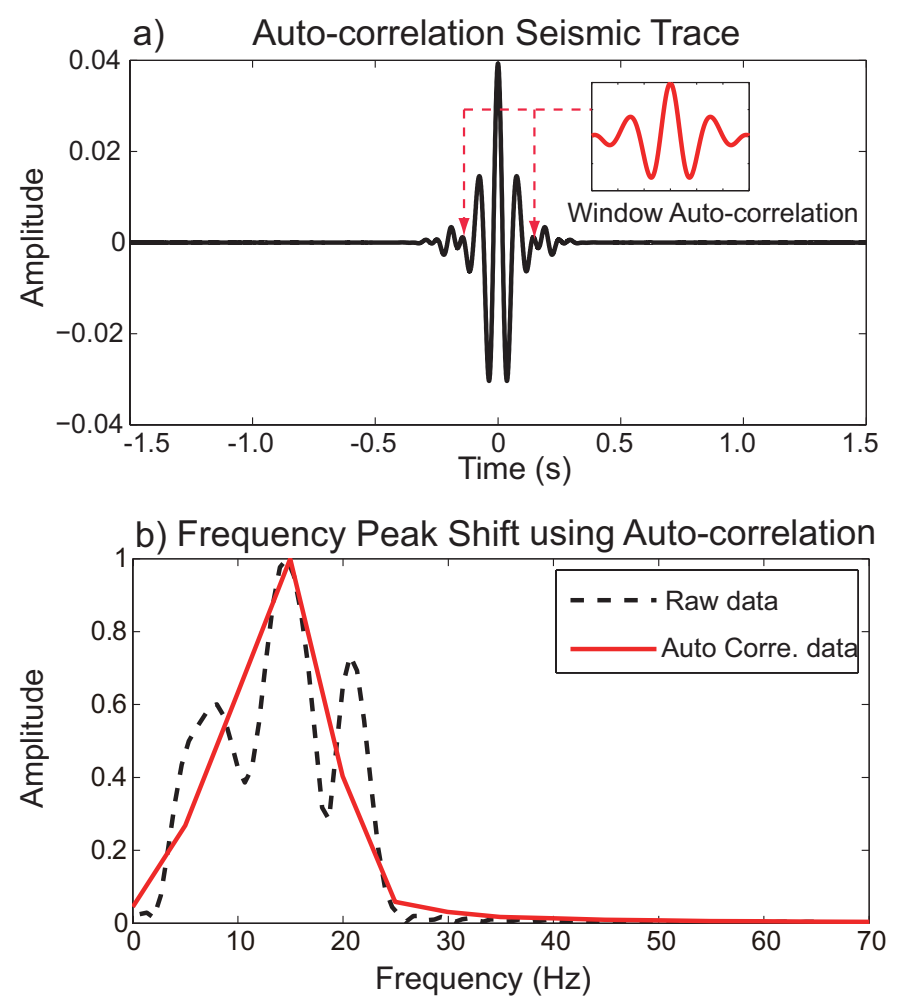

Figure 2. (a) Auto-correlated seismic trace, and (b) the peak-frequency estimated from (a) by windowing the trace around the zero-time lag.

For real data, since the peak-frequency is very sensitive to noise, Gamar et al. (2015) used an auto-correlated trace windowed about the zero-time lag to pick the peak frequency. This increases the robustness of the peak-frequency estimation. Figure 2a shows an auto-correlated observed trace where the amplitude spectrum, shown in Figure $2 b$, is computed only for the part of the signal around the maximum peak of the auto-correlation function. This minimizes the side lobes in the amplitude spectrum, and allows for a more accurate estimate of the peak frequency.

(iv) Compute the residual trace as $\Delta D_{f}\left(\mathbf{x}_{g}, t ; \mathbf{x}_{s}\right)=\frac{1}{K 2} \dot{D}\left(\mathbf{x}_{g}, t ; \mathbf{x}_{s}\right)^{o b s} \Delta f$, which is then backward propagated using equation A.9.

(v) Use equation 14 to compute the gradient $\gamma(\mathbf{x})$.

(vi) Estimate the step-length $\alpha$ using any backtracking line-search method and update the $\eta$ model using the iterative steepest descent method.

$$
\eta^{(k+1)}=\eta^{(k)}-\alpha \overbrace{\sum_{g} \Delta f \frac{\partial \Delta f}{\partial \eta(\mathbf{x})}}^{\gamma(\mathbf{x})=\text { gradient }},
$$

where $\alpha$ is the step-length at the $k$-th iteration. The update for the relaxation ratio $\eta$ is then mapped to $\mathrm{Q}_{s}$ using equation 4 . A more efficient algorithm is to use a preconditioned conjugate gradient method. 

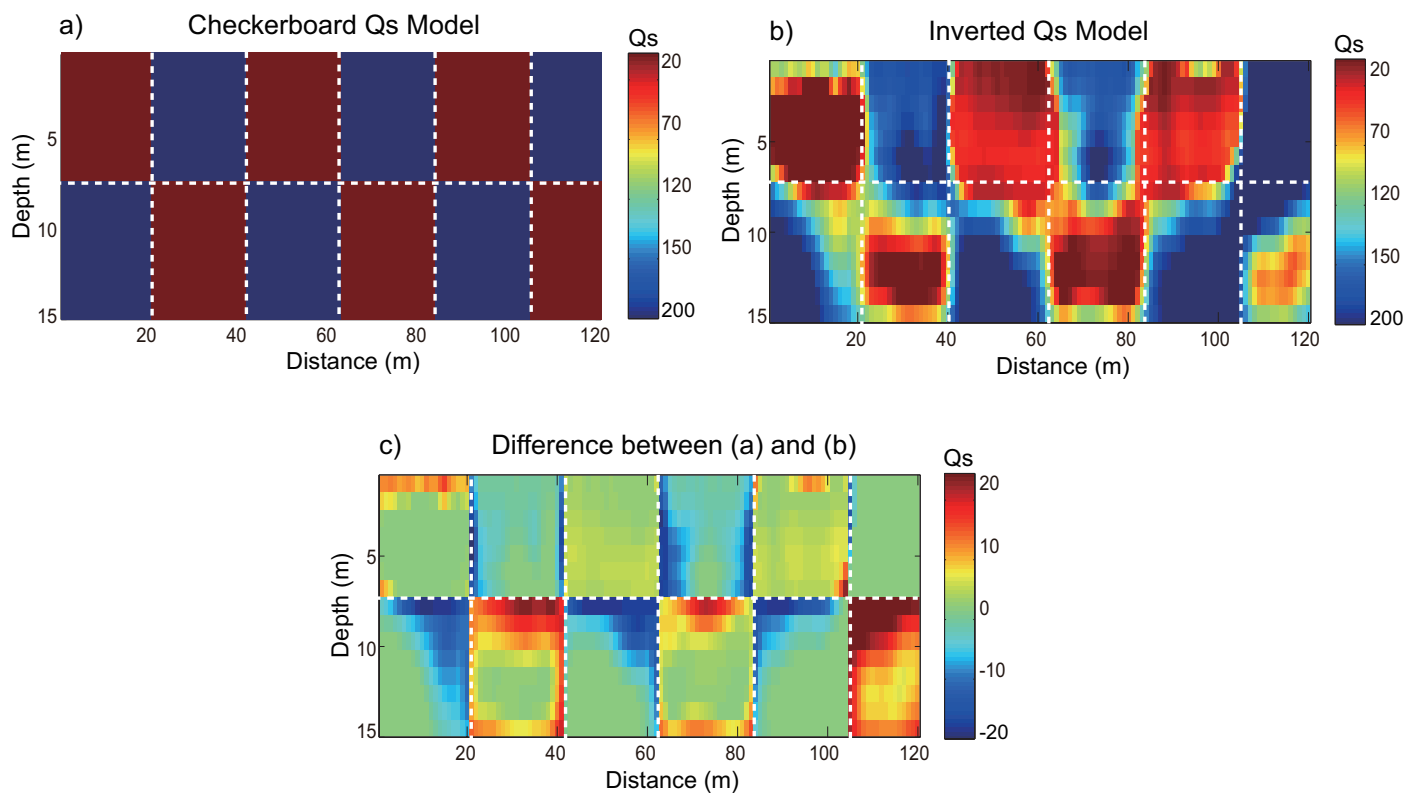

Figure 3. (a) True checkerboard $\mathrm{Q}_{s}$ model, (b) the $\mathrm{Q}_{s}$ tomogram computing by $\mathrm{WQ}_{s}$ and (c) the difference between the true and inverted $\mathrm{Q}_{s}$ models.

\section{NUMERICAL TESTS}

The effectiveness of the $\mathrm{WQ}_{s}$ method is now demonstrated with synthetic and field data examples. The synthetic examples are for two models with strong attenuation: (1) a checkerboard $\mathrm{Q}_{s}$ model to assess the resolution of the method, and (2) a complex near-surface model where there are shallow $\mathrm{Q}_{s}$ anomalies. We also compare the accuracy of the $\mathrm{WQ}_{s}$ tomogram with that computed by full waveform inversion. The field data example is on a seismic data set collected near the Qademah fault, which is $40 \mathrm{~km}$ north of the KAUST campus. In the synthetic examples, the observed data are generated by a 2D staggered-grid solution of the viscoelastic wave equation in equation 3 . The P-wave velocity is estimated from the $\mathrm{S}$-wave velocity using the empirical relationship $\mathrm{V}_{p}=\sqrt{3} \mathrm{~V}_{s}$. In all the numerical examples, the starting $\mathrm{Q}_{s}$ tomogram is taken to be homogeneous with $\mathrm{Q}_{s}=1000$.

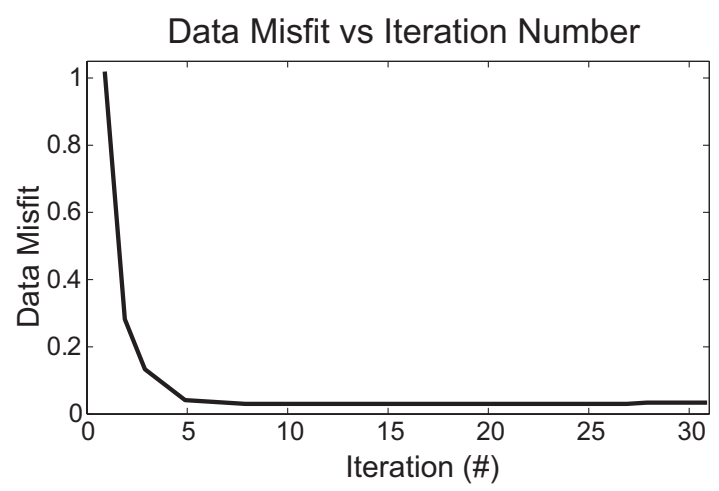

Figure 4. Normalized misfit plotted against iteration number for the $\mathrm{WQ}_{s}$ method applied to the checkerboard data. The Y-axis represents the normalized frequency-shift data residual. 

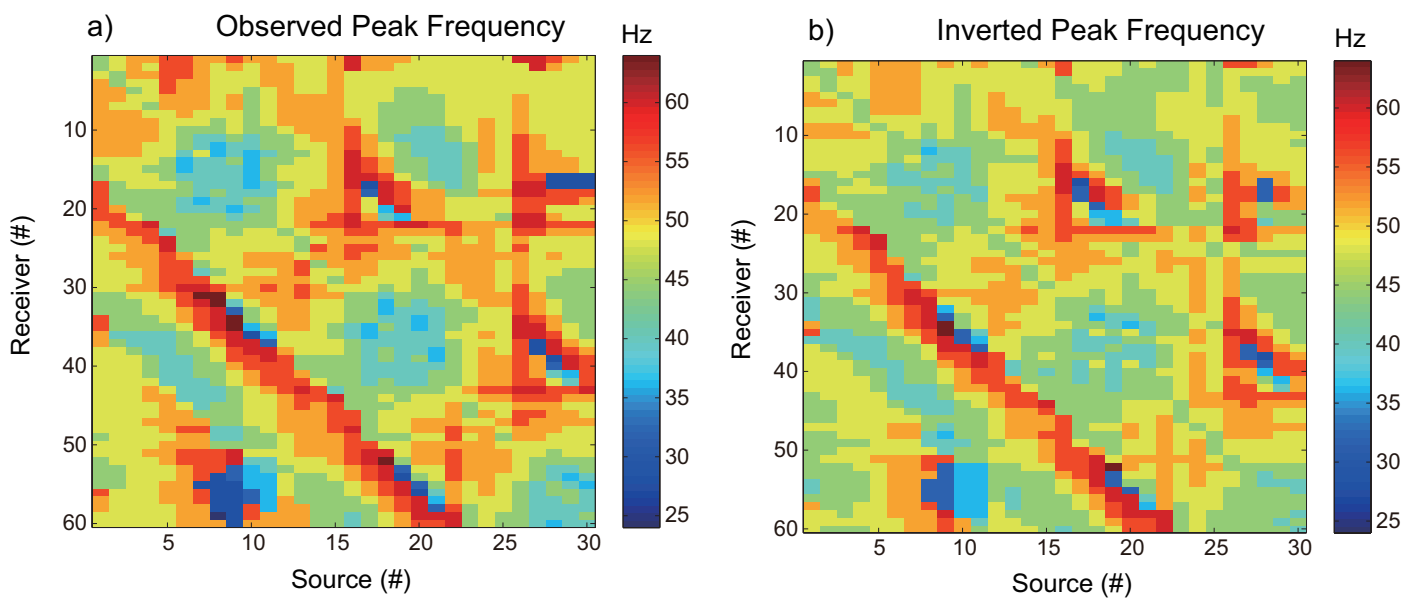

Figure 5. The peak-frequencies for different source-receiver pairs in the (a) observed and (b) predicted data after WQs.

\subsection{Checkerboard Model}

Viscoelastic shot gathers are computed for the checkerboard $\mathrm{Q}_{s}$ model, shown in Figure $3 \mathrm{a}$, to assess the resolution capabilities of the WQ $\mathrm{Q}_{s}$ method. The background S-wave velocity is $800 \mathrm{~m} / \mathrm{s}$. To construct the checkerboard perturbations, low- $\mathrm{Q}_{s}$ anomalies are embedded within the model, where the $\mathrm{Q}_{s}$ model values are changed by 20 and 200. A 40-Hz Ricker wavelet is used as the source function to generate the observed data. The grid spacing and time sampling intervals for the $2 \mathrm{D}$ viscoelastic finite-difference algorithm are $1 \mathrm{~m}$ and $0.03 \mathrm{~ms}$, respectively. The checkerboard model has 6 rectangles where each of the anomalies has an area of $20 \times 7.5 \mathrm{~m}^{2}$. There are 30 vertical-component shot gathers with 60 receivers located every $2 \mathrm{~m}$ on the surface.

The inverted $\mathrm{Q}_{s}$ tomogram is shown in Figure $3 \mathrm{~b}$ where the white dashed lines depict the $\mathrm{Q}_{s}$ interfaces. This tomogram indicates that the $\mathrm{WQ}_{s}$ method is able to estimate the $\mathrm{Q}_{s}$ anomalies with acceptable accuracy, where the resolution decreases with depth and at the edges of the tomogram (Figure 3c) because of the absence of strong surface-wave wavepaths at the edges and at deeper depths. The normalized data misfit values are plotted against iteration number in Figure 4 and the observed and final predicted traces after $\mathrm{WQ}_{s}$ inversion are shown in Figure 5. These results show that the $\mathrm{WQ}_{s}$ method is characterized by robust convergence and acceptable accuracy for the checkerboard model.

\subsection{Complex Velocity Model}

We now compare the performance of $\mathrm{WQ}_{s}$ against that of FWI for the complex near-surface $\mathrm{V}_{s}$ and $\mathrm{Q}_{s}$ models shown in Figure 6. A smooth version of the true S-wave velocity model, shown in Figure 6c, is used as the background velocity model for WQ $\mathrm{W}_{s}$ and FWI. The grid spacing and time sampling intervals for the 2D viscoelastic finite-difference algorithm are $1 \mathrm{~m}$ and $0.025 \mathrm{~ms}$, respectively, and the center frequency 

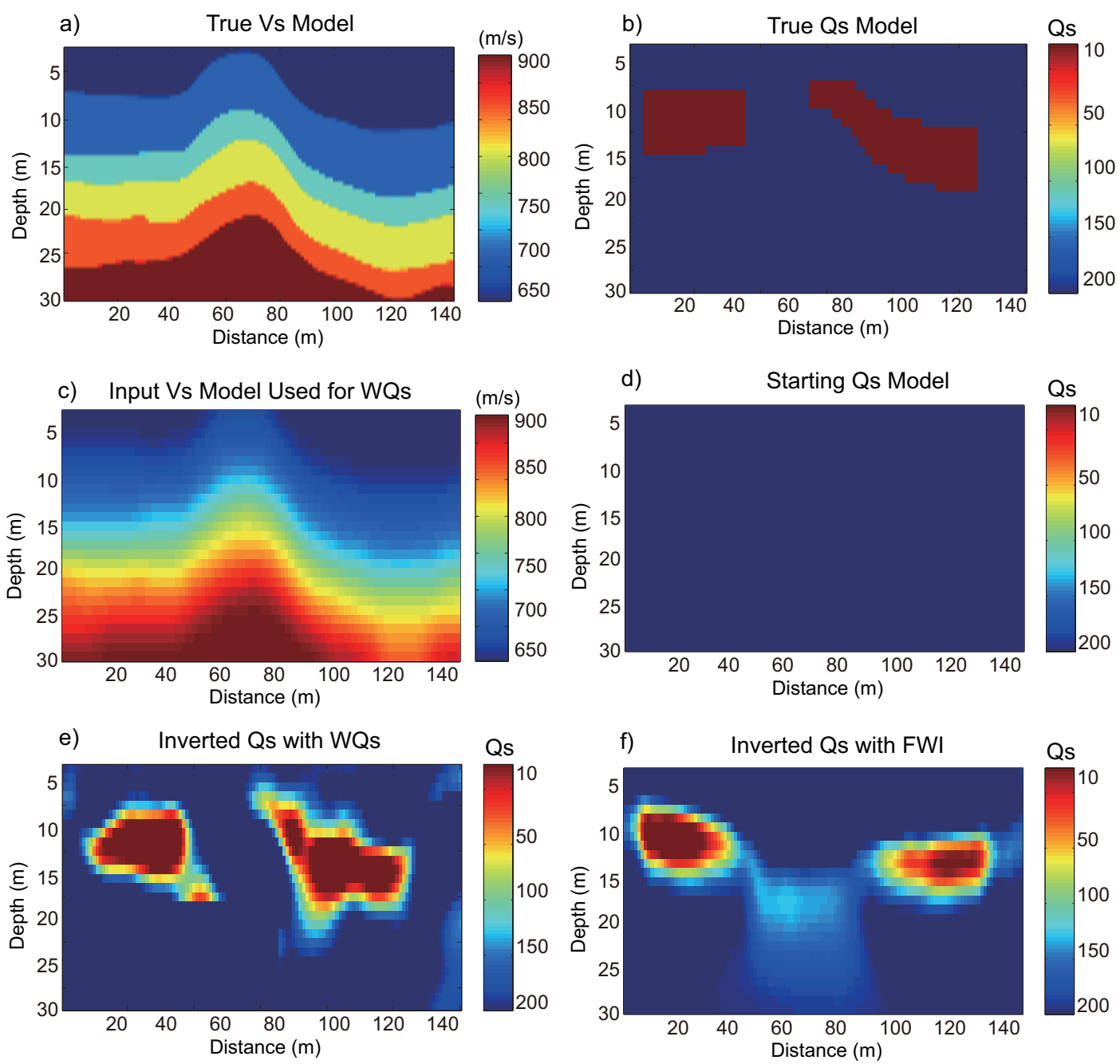

Figure 6. (a) True velocity and (b) $\mathrm{Q}_{s}$ models used for generating the observed data, (c) background $\mathrm{V}_{s}$ model used for $\mathrm{WQ}_{s}$ inversion, (d) starting $\mathrm{Q}_{s}$ model, (e) the $\mathrm{Q}_{s}$ tomogram computed by $\mathrm{WQ}_{s}$ and (f) the $\mathrm{Q}_{s}$ tomogram computed by FWI.

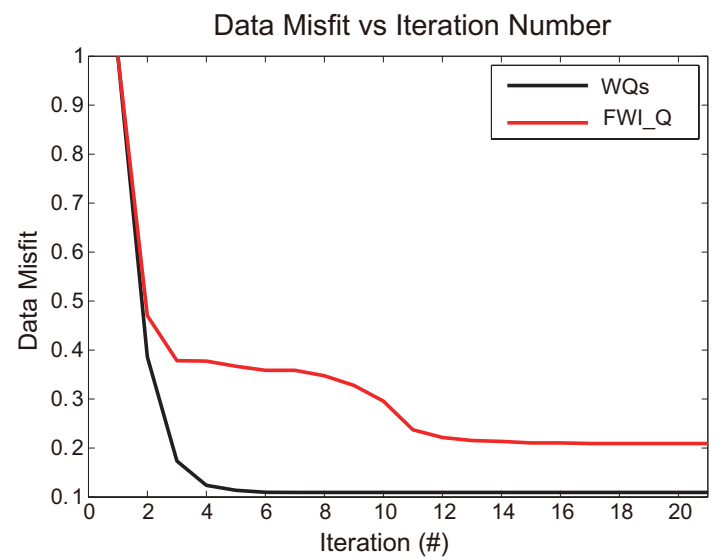

Figure 7. Data misfit plotted against iteration number for the $\mathrm{WQ}_{s}$ and FWI methods applied to data associated with the Figure $6 \mathrm{a}$ and $6 \mathrm{~b}$ models. The $\mathrm{Y}$-axis represents the normalized frequency-shift residual. 

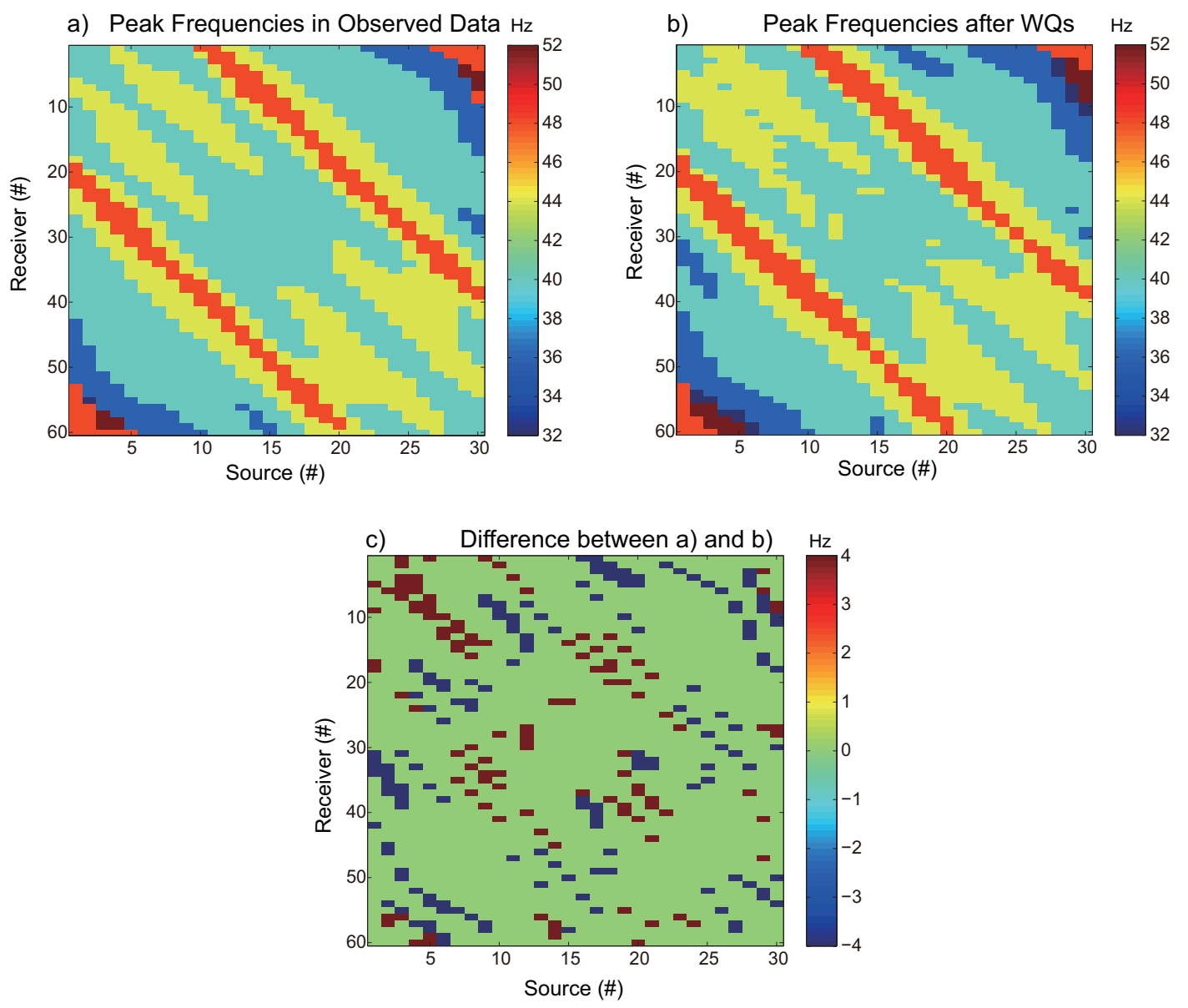

Figure 8. The peak-frequencies for different source-receiver pairs in (a) the observed data, (b) the predicted data after $\mathrm{WQ}_{s}$, and (c) their differences.

of the source wavelet is $35 \mathrm{~Hz}$. The observed data are generated by 40 shots evenly distributed on the surface and the data are recorded by 70 receivers every $2 \mathrm{~m}$ on the surface. The initial $\mathrm{Q}_{s}$ model is a homogeneous half space (Figure 6d).

The $\mathrm{Q}_{s}$ tomograms from the $\mathrm{WQ}_{s}$ and FWI methods after 21 iterations are shown in Figures 6e and 6f, respectively. It is obvious that the $\mathrm{WQ}_{s}$ tomogram is more accurate than the FWI tomogram for the same number of iterations. In addition, Figure 7 shows that after 21 iterations, the normalized $\mathrm{WQ}_{s}$ residual (black line) is less than the FWI waveform residual (red line). Figure 8 reveals that the predicted peak frequencies are very close to the actual ones for many source-receiver pairs.

\subsection{Field Data Example}

The $\mathrm{WQ}_{s}$ method is now tested on a near-surface field data set recorded over the Qademah fault. Here, the location of the field experiment is shown in Figure 9 and the red dashed line denotes the Qademah fault aligned along the north-south direction. The blue solid line is the survey line where 60 receivers are located at $10 \mathrm{~m}$ intervals with 60 shots positioned at $10 \mathrm{~m}$ intervals. Before the $\mathrm{WQ}_{s}$ inversion, the following processing steps are applied to the data: 


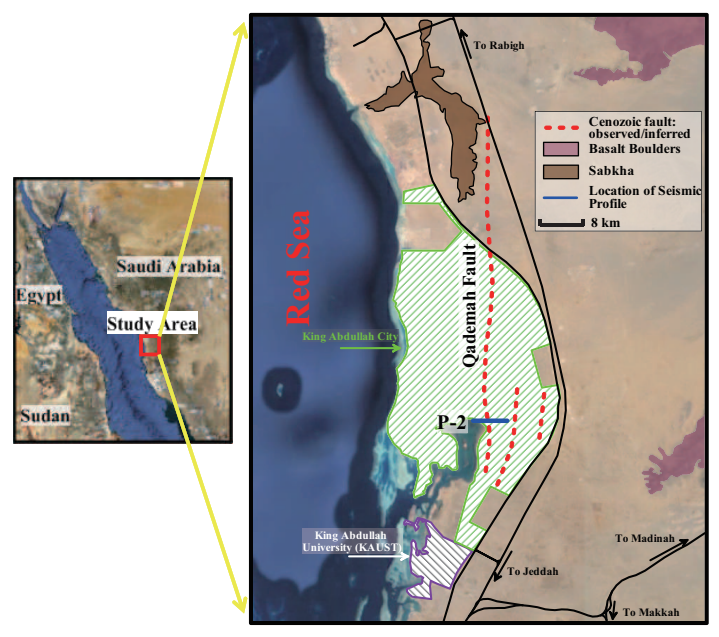

Figure 9. The seismic survey line across the Qademah fault.

- A 5-20 Hz bandpass filter is applied to the data to remove noise.

- The body-wave arrivals are muted and only the Rayleigh-wave arrivals are kept in the input shot gathers.

- The recorded data are corrected from 3D to $2 \mathrm{D}$ format by scaling the amplitudes by $\sqrt{t}$ to approximate geometrical spreading. A phase correction is applied to the traces by multiplying the spectrum of the observed seismogram with the filter $\sqrt{i / \omega}$ (Zhou et al. 1995).

- The data are Wiener filtered to transform the original wavelet to a Ricker wavelet with a $10 \mathrm{~Hz}$ peak-frequency. This bandwidth is based on the frequency content of the data where most of the signal is concentrated between 5-20 Hz. The surface waves are windowed for each observed and predicted shot gather.

A final processed CSG at the source location $\mathrm{X}=0 \mathrm{~m}$ is shown in Figure 10. The first-arrival traveltimes are picked in all the CSGs and inverted using ray-based traveltime tomography to obtain the P-wave velocity model shown in Figure 11a. The S-wave tomogram is shown in Figure 11b, which is obtained using the surface-wave dispersion inversion method of Li and Schuster ( 2016). These velocity models are used as the background velocity models for $\mathrm{WQ}_{s}$. For $\mathrm{WQ}_{s}$, the starting $\mathrm{Q}_{s}$ model is taken to be homogeneous with $\mathrm{Q}_{s}=1000$ and the inverted $\mathrm{Q}_{s}$ tomogram is shown in Figure 12a. There is reasonable geological agreement between the $\mathrm{S}$-wave velocity and the $\mathrm{Q}_{s}$ tomograms

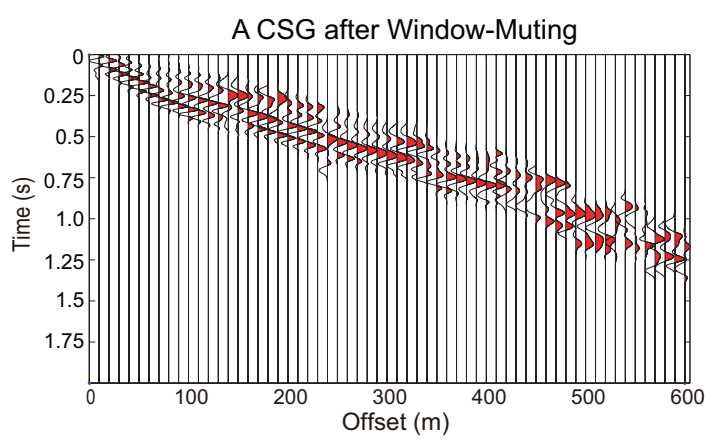

Figure 10. A common shot gather recorded by the Qademah field experiment after applying all the processing steps. 

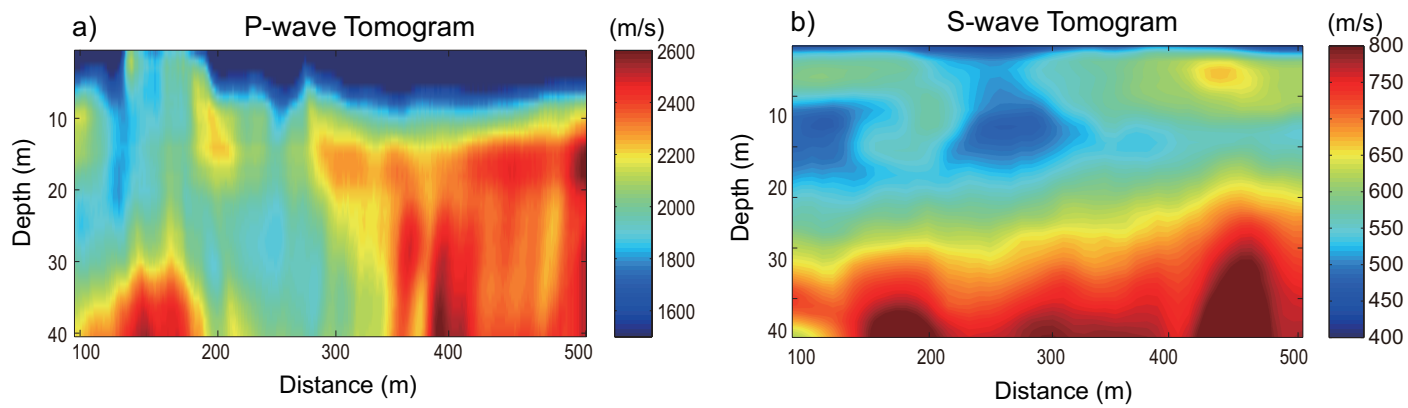

Figure 11. (a) P-wave tomogram with ray tracing tomography and (b) S-wave velocity tomogram by wave equation dispersion inversion (Li et al. 2017).

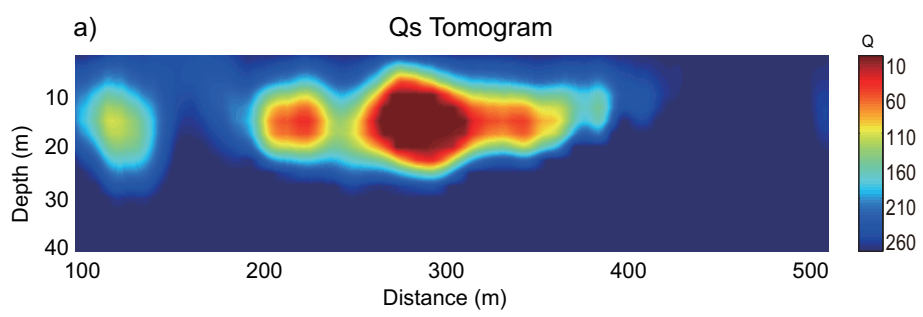

b)

Vp/Vs Ratio
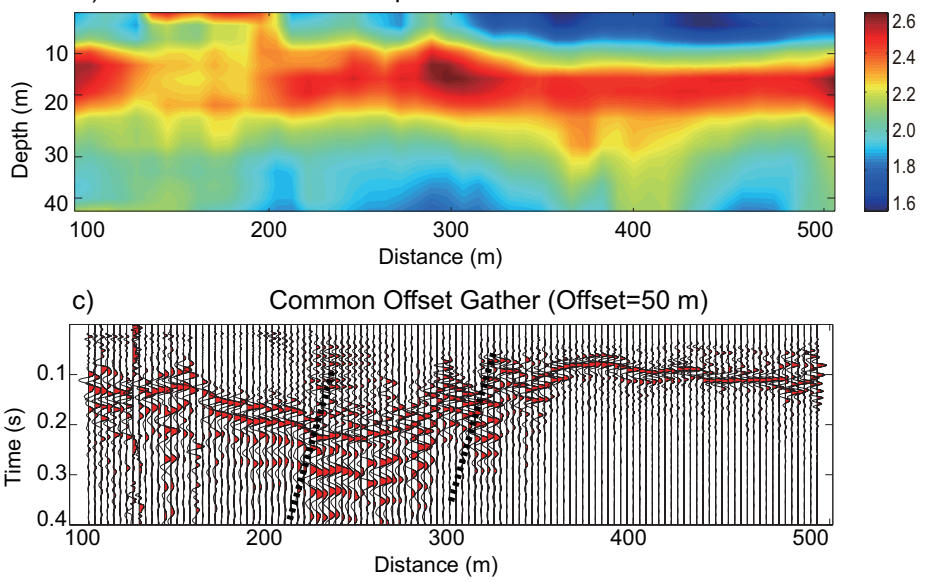

Figure 12. (a) $\mathrm{WQ}_{s}$ tomogram, (b) ratio $\left(\mathrm{V}_{p} / \mathrm{V}_{s}\right)$ computed from a) and b), and (c) common offset gather (offset=50 m) profile after data processing.

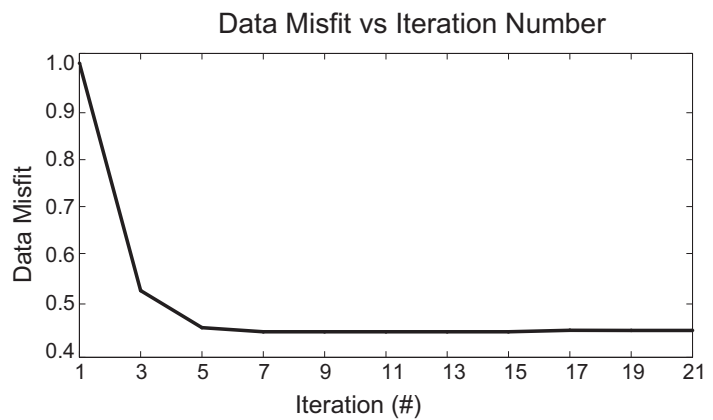

Figure 13. Data misfit values plotted against iteration number for the $\mathrm{WQ}_{s}$ method. The Y-axis represents the normalized frequency-shift data residual. 

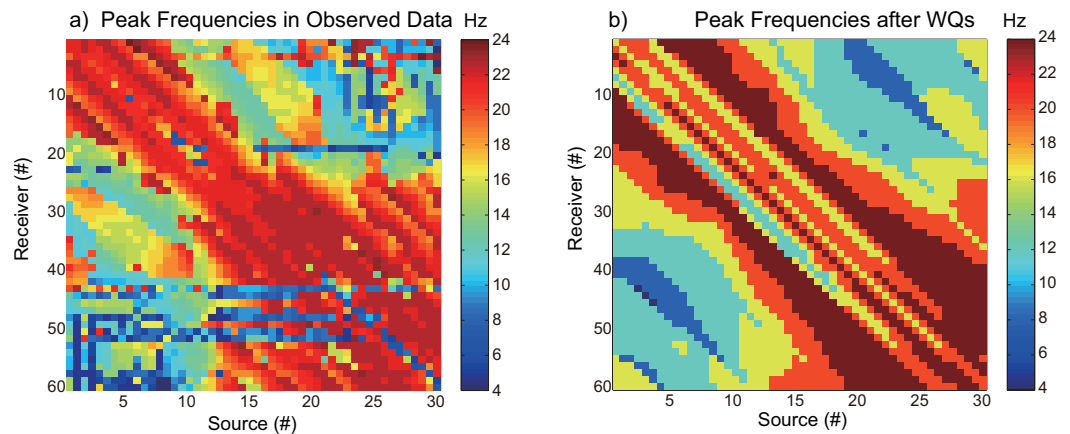

Figure 14. The peak frequencies for different source-receiver pairs for the a) observed and b) inverted data.

(Figure 11b and Figure 12a). The high attenuation regions in the $\mathrm{Q}_{s}$ tomogram (low $\mathrm{Q}_{s}$ values) correspond to the low $\mathrm{S}$-wave velocity regions (De Meersman 2013; Zhu \& Harris 2015). Previous work by Zhang et al. ( 2015) demonstrated that areas with high $\mathrm{V}_{p} / \mathrm{V}_{s}$ ratios tend to have low $\mathrm{Q}$ values (or high attenuation) while the variation of $\mathrm{Q}_{p}$ and $\mathrm{Q}_{s}$ is the same. We calculate the $\mathrm{V}_{p} / \mathrm{V}_{s}$ ratio using the tomograms in Figure 11 and the ratio is shown in Figure $12 \mathrm{~b}$. It can be seen from this figure that areas with high $\mathrm{V}_{p} / \mathrm{V}_{s}$ ratio have low $\mathrm{Q}_{s}$ values, as seen in Figure 12a.

As a final check, the inverted $\mathrm{Q}_{s}$ tomogram is compared to a common offset gather (COG) profile (Hanafy et al. 2015). Figure 12c shows a COG profile using the processed data for an offset of $50 \mathrm{~m}$. The black dashed line in this figure shows the location of the fault which is between $250-300 \mathrm{~m}$. The locations of the $\mathrm{Q}_{s}$ anomalies in the $\mathrm{WQ}_{s}$ tomogram and the low-velocity area in the S-wave tomogram are consistent with the location of the fault, as indicated in the COG profile. Figure 13 shows that the data misfit is reduced by $45 \%$ after 17 iterations, and there is reasonable agreement between the peak frequencies of the observed and the predicted traces as shown in Figures 14a and $14 \mathrm{~b}$.
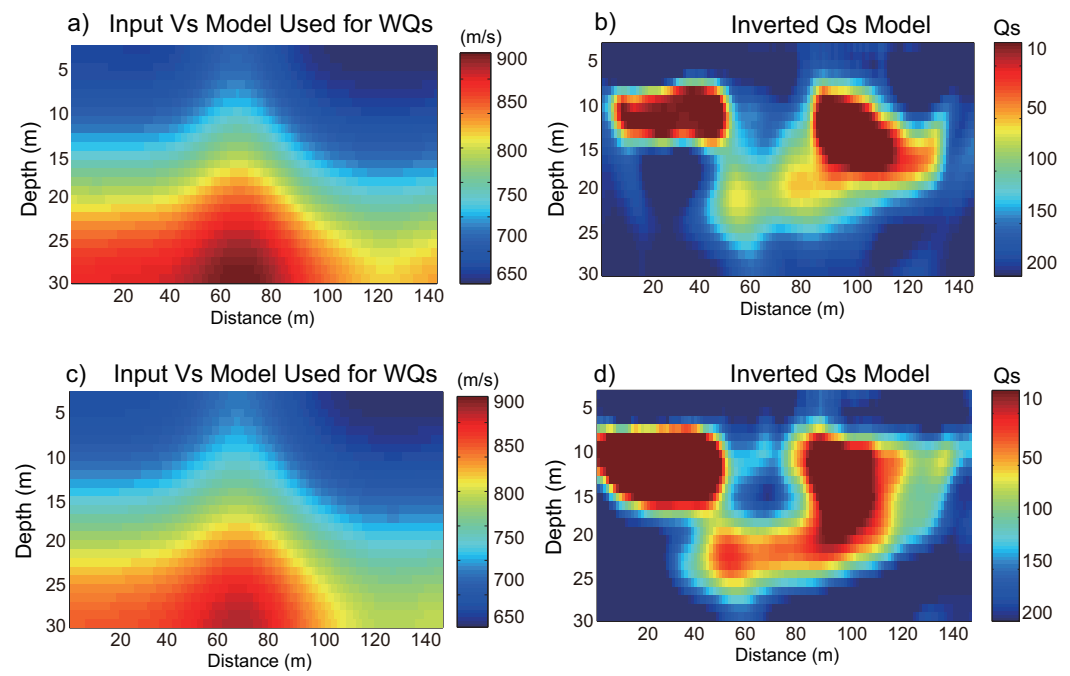

Figure 15. $\mathrm{WQ}_{s}$ tomograms using different background velocity models. (a) and (c) are $20 \%$ and $40 \%$ smoothed versions of the true velocity model. (b) and (d) are the corresponding $\mathrm{WQ}_{s}$ tomograms. 

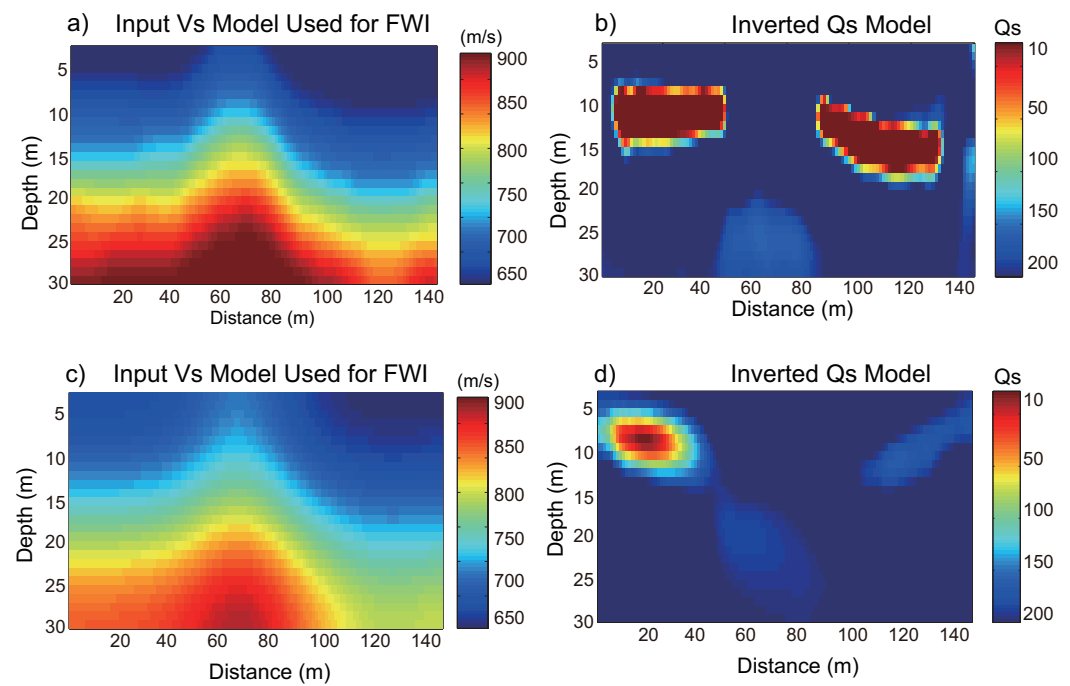

Figure 16. The $\mathrm{Q}_{s}$ tomograms obtained from FWI using different background velocity models, (a) and (c) are the starting velocity models obtained by smoothing the actual velocity model by a filter that is $10 \%$ and $40 \%$ the size of the model, respectively., (b) and (d) are the corresponding $\mathrm{Q}_{s}$ tomograms.

a) Vs Profiles for Different Starting Models

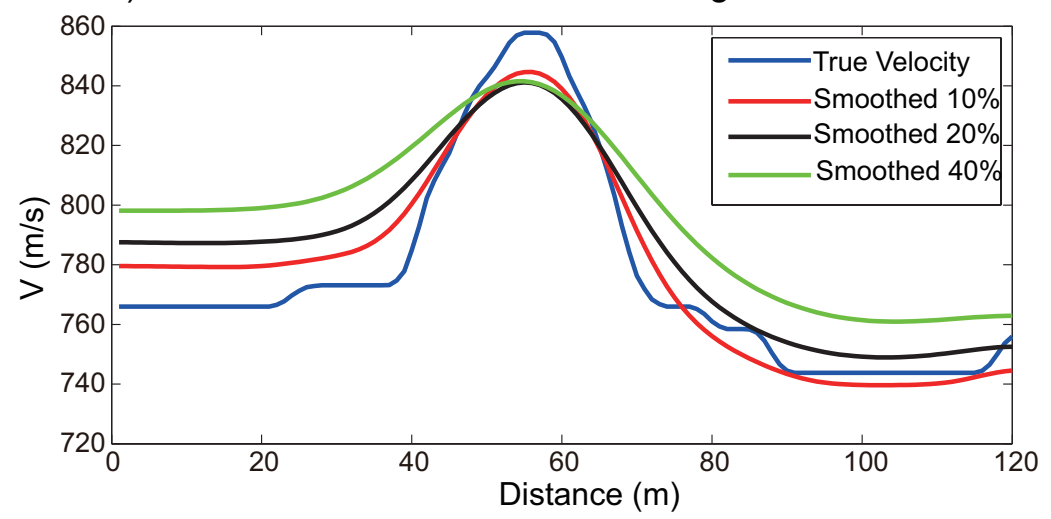

b) WQs and FWI Profiles for Different Starting Models

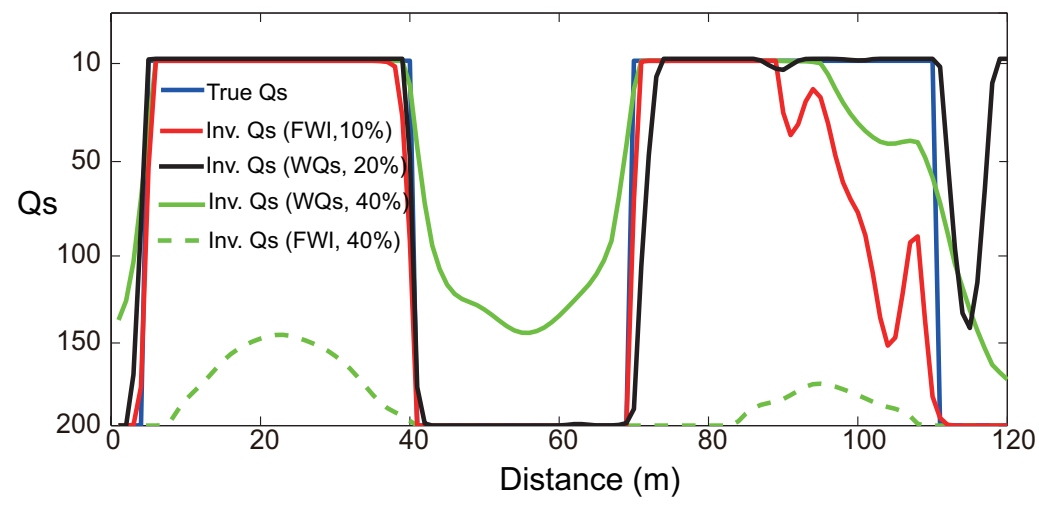

Figure 17. (a) Different background velocity models and (b) the corresponding $\mathrm{WQ}_{s}$ and FWI horizontal profiles at the depth of $15 \mathrm{~m}$. 


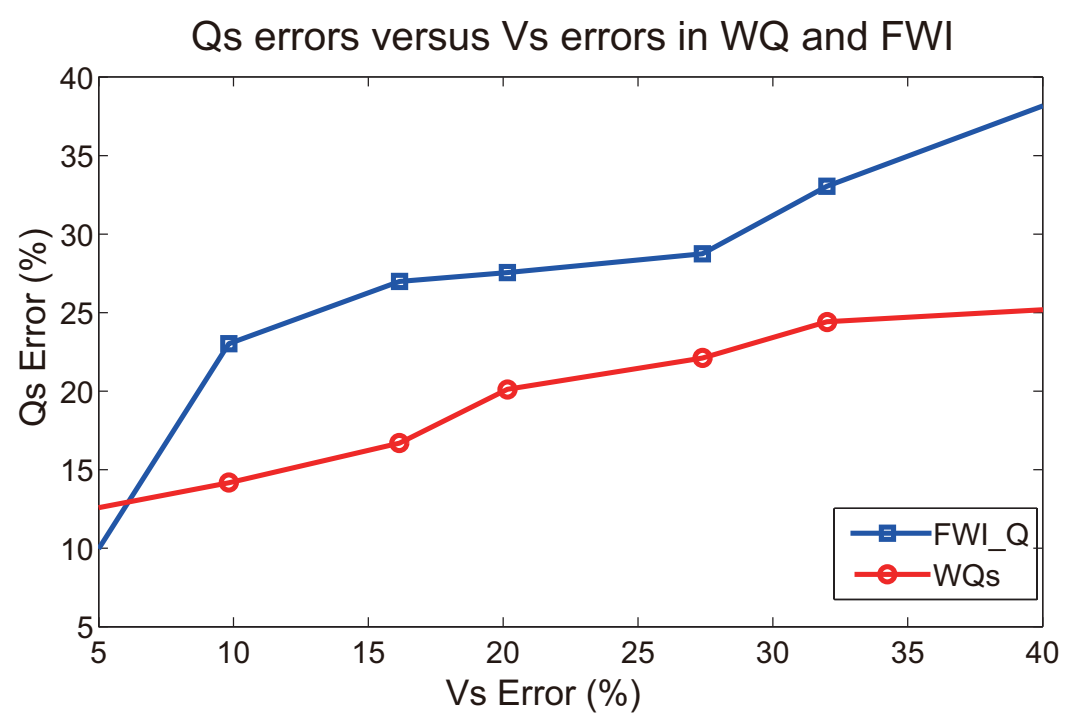

Figure 18. $\mathrm{Q}_{s}$ global errors versus $\mathrm{V}_{s}$ global errors for WQ and FWI.

\section{DISCUSSION}

Both synthetic and field-data examples demonstrate that the $\mathrm{WQ}_{s}$ method can be used to invert for smoothed approximations to the $Q_{s}$ models. According to the discussion in Dutta and Schuster ( 2016), the reliability of any Q tomogram is dependent on the accuracy of the background velocity model (Liao \& McMechan 1995; Kamei \& Pratt 2008; Virieux \& Operto 2009). If the background velocity has significant errors, it will be lead to large inaccuracies in the inverted $\mathrm{Q}_{s}$ tomogram. To analyze this problem, we use the smoothed background velocity to increase the error between the input background velocity model and the true velocity model in Figure 6a. The different smoothed velocity models (20\% and 40\%) are shown in Figures $15 \mathrm{a}$ amd $15 \mathrm{c}$. The errors in the $\mathrm{WQ}_{s}$ tomograms in Figures $15 \mathrm{~b}$ and $15 \mathrm{~d}$ increase when the errors in the input background velocity model increase. However, the shape and position of the low $\mathrm{Q}_{s}$ anomalies are located with reasonable accuracy in the tomograms.

We also use the smoothed input velocity model to evaluate the sensitivity of the FWI method to different starting velocity models. The different background velocity models are shown in Figures 16a amd 16c. If the background velocity model is only smoothed by $10 \%$ (Figure 16a), the FWI method provides a reasonably accurate $\mathrm{Q}_{s}$ tomogram. However, if the smoothing scale is $40 \%$, the FWI method produces a $\mathrm{Q}_{s}$ tomogram that has unacceptable inaccuracies. It is evident from these tomograms that the $\mathrm{WQ}_{s}$ approach should be preferred over FWI because the $\mathrm{WQ}_{s}$ tomogram is less sensitive to errors in the velocity model as shown in Figures 17 and 18.

In all of our examples, we use the single SLS mechanism for the time-domain viscoelastic wave equation. However, to obtain a constant Q response over a large frequency bandwidth, it is recommended to use at least 3 SLS mechanisms (Emmerich \& Korn 1987; Zhu et al. 2013). The theory of wave-equation Qs inversion using Rayleigh waves, as proposed in this paper, will hold true, irrespective of the type of 
SLS mechanism. However, the derivation of the gradient and the adjoint equations needs to be modified according to the particular damping model.

\section{CONCLUSIONS}

We presented a skeletonized surface-wave wave-equation $\mathrm{Q}_{s}$ inversion method, where the $Q_{s}$ model is found that minimizes the sum of the squared difference $\sum_{i} \Delta f_{i}^{2}$ in the peak frequencies of the observed and the predicted surface-wave arrivals. The gradient for WQ $\mathrm{Q}_{s}$ is derived using the implicit function theorem and is numerically obtained by a zero-lag cross-correlation between the forward propagated viscoelastic source wavefield and the weighted backprojected residuals. Here, the weight is proportional to $\Delta f_{i}$ at the $i t h$ trace. This method does not require a simplifying layered-medium assumption. The limitation of $\mathrm{WQ}_{s}$, however, is a loss of $\mathrm{Q}_{s}$ resolution compared to methods that invert for all the amplitudes in the surface-wave arrivals. Another limitation is that, similar to all $Q_{s}$ inversion methods, it requires an accurate estimation of the $\mathrm{V}_{s}$ model. Therefore, future research should explore the value of simultaneously (or jointly) inverting for both the $\mathrm{V}_{s}$ and $\mathrm{Q}_{s}$ models.

\section{ACKNOWLEDGEMENTS}

The research reported in this publication was supported by the King Abdullah University of Science and Technology (KAUST) in Thuwal, Saudi Arabia. We are grateful to the sponsors of the Center for Subsurface Imaging and Modeling (CSIM) consortium for their financial support. For computer time, this research used the resources of the IT Research Computing Group and the Supercomputing Laboratory at

KAUST. We thank them for providing the computational resources required for carrying out this work.

\section{APPENDIX A: VISCOELASTIC GRADIENT FOR $\eta$}

The gradient for the $\mathrm{WQ}_{s}$ method is now derived (Schuster 2017). In matrix vector notation, equation 3 can be written as 


$$
\left(\begin{array}{cccccccc}
-\rho \frac{\partial}{\partial t} & 0 & \frac{\partial}{\partial x} & 0 & \frac{\partial}{\partial z} & 0 & 0 & 0 \\
0 & -\rho \frac{\partial}{\partial t} & 0 & \frac{\partial}{\partial z} & \frac{\partial}{\partial x} & 0 & 0 & 0 \\
\pi C \frac{\partial}{\partial x} & (\pi C-2 \mu \eta) \frac{\partial}{\partial z} & -\frac{\partial}{\partial t} & 0 & 0 & 1 & 0 & 0 \\
(\pi C-2 \mu \eta) \frac{\partial}{\partial x} & \pi C \frac{\partial}{\partial z} & 0 & -\frac{\partial}{\partial t} & 0 & 0 & 1 & 0 \\
\mu \eta \frac{\partial}{\partial z} & \mu \eta \frac{\partial}{\partial x} & 0 & 0 & -\frac{\partial}{\partial t} & 0 & 0 & 1 \\
\pi F \frac{\partial}{\partial x} & (\pi F-2 \mu H) \frac{\partial}{\partial z} & 0 & 0 & 0 & -\frac{\partial}{\partial t}+E & 0 & 0 \\
(\pi F-2 \mu H) \frac{\partial}{\partial x} & \pi F \frac{\partial}{\partial z} & 0 & 0 & 0 & 0 & -\frac{\partial}{\partial t}+E & 0 \\
\mu H \frac{\partial}{\partial z} & \mu H \frac{\partial}{\partial x} & 0 & 0 & 0 & 0 & 0 & -\frac{\partial}{\partial t}+E
\end{array}\right)\left(\begin{array}{c}
u \\
w \\
\sigma_{x x} \\
\sigma_{z z} \\
\sigma_{x z} \\
r_{x x} \\
r_{z z} \\
r_{x z}
\end{array}\right)=\left(\begin{array}{c}
f_{x x} \\
f_{z z} \\
0 \\
0 \\
0 \\
0 \\
0 \\
0 \\
0 \\
0
\end{array}\right.
$$

or in a more compact form

$$
\mathbf{A}(\mathbf{m}) \mathbf{w}(\mathbf{m})=\mathbf{F}
$$

where

$$
C=\frac{\tau_{\epsilon}^{P}}{\tau_{\sigma}} ; \eta=\frac{\tau_{\epsilon}^{S}}{\tau_{\sigma}} ; E=\frac{-1}{\tau_{\sigma}} ; F=E\left(\frac{\tau_{\epsilon}^{P}}{\tau_{\sigma}}-1\right) ; H=E(\eta-1)
$$

The perturbation of the state variable $\mathbf{w}$ with respect to to the model variable $\mathbf{m}$ is given by $\frac{\partial \mathbf{w}(\mathbf{m})}{\partial \mathbf{m}}$ and can be obtained from

$$
\begin{aligned}
& \mathbf{A}(\mathbf{m}) \mathbf{w}(\mathbf{m})=\mathbf{F}, \\
\Rightarrow & \frac{\partial \mathbf{A}(\mathbf{m})}{\partial \mathbf{m}} \mathbf{w}(\mathbf{m})+\mathbf{A}(\mathbf{m}) \frac{\partial \mathbf{w}(\mathbf{m})}{\partial \mathbf{m}}=\mathbf{0}, \\
\Rightarrow & \frac{\partial \mathbf{w}(\mathbf{m})}{\partial \mathbf{m}}=-\mathbf{A}^{-1}(\mathbf{m}) \frac{\partial \mathbf{A}(\mathbf{m})}{\partial \mathbf{m}} \mathbf{w}(\mathbf{m}) .
\end{aligned}
$$

The connective function in equation 9 can also be written as

$$
\dot{\Phi}_{\Delta f}=<\mathbf{A}_{r} \mathbf{w}_{f}\left(\mathbf{x}_{g}, t ; \mathbf{x}_{s}\right), \dot{\mathbf{w}}_{f-\Delta f}\left(\mathbf{x}_{g}, t ; \mathbf{x}_{s}\right)^{o b s}>
$$

Here $\left\langle\mathbf{u}, \mathbf{v}>\right.$ represents the inner product between the vectors $\mathbf{u}$ and $\mathbf{v}$ and $\mathbf{A}_{r}$ is a sampling operator that samples the wavefield at the receiver locations while the adjoint of it, $\mathbf{A}_{r}^{*}$, sprays the recorded data from the receiver coordinates to the model coordinates. $\mathbf{w}_{f}$ denotes the predicted event for a given background $\mathrm{Q}_{s}$ model recorded at the receiver location $\mathbf{x}_{g}$ due to a source excited at time $\mathrm{t}=0$ and at location $\mathbf{x}_{s}$ while $\mathbf{w}_{f-\Delta f}^{\text {obs }}$ denotes the same event in the observed data.

From equation 6 , the misfit function for $\mathrm{WQ}_{s}$ is given by

$$
\epsilon=\frac{1}{2} \sum_{g} \sum_{s} \Delta f\left(\mathbf{x}_{g}, \mathbf{x}_{s}\right)^{2}
$$


and the gradient can be written as

$$
\begin{aligned}
\frac{\partial \epsilon}{\partial \eta} & =\sum_{s} \sum_{g} \frac{\frac{\partial \dot{\Phi}}{\partial \eta(\mathbf{x})}}{\frac{\partial \dot{\Phi}}{\partial \Delta f}} \Delta f\left(\mathbf{x}_{g}, \mathbf{x}_{s}\right), \\
& =-\sum_{s} \sum_{g} \frac{1}{\mathbf{K}} \frac{\partial \dot{\Phi}}{\partial \eta(\mathbf{x})} \Delta f\left(\mathbf{x}_{g}, \mathbf{x}_{s}\right), \\
& =\sum_{s} \sum_{g} \frac{1}{\mathbf{K}} \frac{\partial}{\partial \eta}<\mathbf{A}_{r} \mathbf{w}_{f}\left(\mathbf{x}, t ; \mathbf{x}_{s}\right), \dot{\mathbf{w}}_{f-\Delta f}\left(\mathbf{x}_{g}, t ; \mathbf{x}_{s}\right)^{o b s}>\Delta f\left(\mathbf{x}_{g}, \mathbf{x}_{s}\right),(\text { using equation A.5 }) \\
& =\sum_{s} \sum_{g}<\mathbf{A}_{r} \frac{\partial \mathbf{w}_{f}\left(\mathbf{x}, t ; \mathbf{x}_{s}\right)}{\partial \eta}, \dot{\mathbf{w}}_{f-\Delta f}\left(\mathbf{x}_{g}, t ; \mathbf{x}_{s}\right)^{o b s} \Delta f\left(\mathbf{x}_{g}, \mathbf{x}_{s}\right) \frac{1}{\mathbf{K}}>, \\
& =-\sum_{s} \sum_{g}<\mathbf{A}_{r} \mathbf{A}-1 \frac{\partial \mathbf{A}}{\partial \eta} \mathbf{w}_{f}\left(\mathbf{x}, t ; \mathbf{x}_{s}\right), \dot{\mathbf{w}}_{f-\Delta f}\left(\mathbf{x}_{g}, t ; \mathbf{x}_{s}\right)^{o b s} \Delta f\left(\mathbf{x}_{g}, \mathbf{x}_{s}\right) \frac{1}{\mathbf{K}}>,(\text { using equation A.4 }) \\
& =-\sum_{s}<\frac{\partial \mathbf{A}}{\partial \eta} \mathbf{w}_{f}\left(\mathbf{x}, t ; \mathbf{x}_{s}\right),\left(\mathbf{A}^{-1}\right)^{*} \sum_{g}\left(\mathbf{A}_{r}^{*} \dot{\mathbf{w}}_{f-\Delta f}\left(\mathbf{x}_{g}, t ; \mathbf{x}_{s}\right)^{o b s} \Delta f\left(\mathbf{x}_{g}, \mathbf{x}_{s}\right) \frac{1}{\mathbf{K}}\right)>, \\
& =-\sum_{s}<\frac{\partial \mathbf{A}}{\partial \eta} \mathbf{w}_{f}\left(\mathbf{x}, t ; \mathbf{x}_{s}\right), \mathbf{w}^{*}\left(\mathbf{x}, t ; \mathbf{x}_{s}\right)>
\end{aligned}
$$

Here,

$$
\mathbf{K}=\left(\begin{array}{c}
K_{1} \\
K_{2} \\
K_{3} \\
K_{4} \\
K_{5} \\
K_{6} \\
K_{7} \\
K_{8}
\end{array}\right)=\left(\begin{array}{c}
\int u\left(\mathbf{x}_{g}, t ; \mathbf{x}_{s}\right) \ddot{u}\left(\mathbf{x}_{g}, t ; \mathbf{x}_{s}\right)^{o b s} d t \\
\int w\left(\mathbf{x}_{g}, t ; \mathbf{x}_{s}\right) \ddot{w}\left(\mathbf{x}_{g}, t ; \mathbf{x}_{s}\right)^{o b s} d t \\
\int \sigma_{x x}\left(\mathbf{x}_{g}, t ; \mathbf{x}_{s}\right) \ddot{\sigma}_{x x}\left(\mathbf{x}_{g}, t ; \mathbf{x}_{s}\right)^{o b s} d t \\
\int \sigma_{z z}\left(\mathbf{x}_{g}, t ; \mathbf{x}_{s}\right) \ddot{\sigma}_{z z}\left(\mathbf{x}_{g}, t ; \mathbf{x}_{s}\right)^{o b s} d t \\
\int \sigma_{x z}\left(\mathbf{x}_{g}, t ; \mathbf{x}_{s}\right) \ddot{\sigma}_{x z}\left(\mathbf{x}_{g}, t ; \mathbf{x}_{s}\right)^{o b s} d t \\
\int r_{x x}\left(\mathbf{x}_{g}, t ; \mathbf{x}_{s}\right) \ddot{r}_{x x}\left(\mathbf{x}_{g}, t ; \mathbf{x}_{s}\right)^{o b s} d t \\
\int r_{z z}\left(\mathbf{x}_{g}, t ; \mathbf{x}_{s}\right) \ddot{r}_{z z}\left(\mathbf{x}_{g}, t ; \mathbf{x}_{s}\right)^{o b s} d t \\
\int r_{x z}\left(\mathbf{x}_{g}, t ; \mathbf{x}_{s}\right) \ddot{r}_{x z}\left(\mathbf{x}_{g}, t ; \mathbf{x}_{s}\right)^{o b s} d t
\end{array}\right)
$$

and $\mathbf{w}^{*}=\left(\hat{u}, \hat{w}, \hat{\sigma}_{x x}, \hat{\sigma}_{z z}, \hat{\sigma}_{x z}, \hat{r}_{x x}, \hat{r}_{z z}, \hat{r}_{x z}\right)$ is the adjoint-state variable of $\mathbf{w}=\left(u, w, \sigma_{x x}, \sigma_{z z}, \sigma_{x z}, r_{x x}, r_{z z}, r_{x z}\right)$ which is calculated by a finite-difference solution for the adjoint-state equations of the viscoelastic wave equation. For Rayleigh waves, since the recorded data are vertical-component particle-velocity traces $w\left(\mathbf{x}_{g}, t ; \mathbf{x}_{s}\right)^{\text {obs }}$, the adjoint viscoelastic equation can be derived from the forward-modeling equation A.1. The adjoint of $f(x) \frac{\partial}{\partial x}$ is $-\frac{\partial[f(x)]}{\partial x}$. In this case, finite support implies zero boundary conditions at infinity (Schuster 2017), so 
the adjoint of the viscoelastic equations can be written as

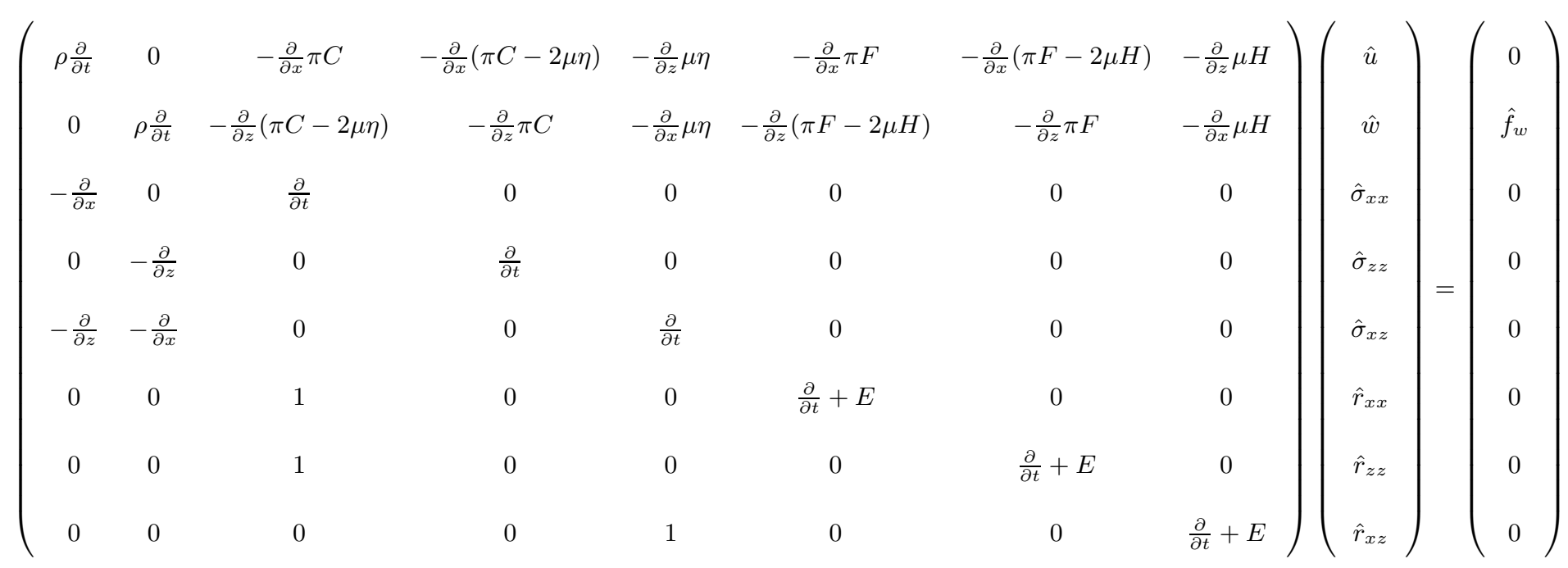

Here, $\hat{f}_{w}$ is the residual trace that is backpropagated at every iteration and is given by

$$
\hat{f}_{w}=\frac{1}{K_{2}} \dot{w}\left(\mathbf{x}_{g}, t ; \mathbf{x}_{s}\right)^{o b s} \Delta f\left(\mathbf{x}_{g}, \mathbf{x}_{s}\right) \text {. }
$$

Using equation A.3, we can get

$$
\frac{\partial \mathbf{A}}{\partial \eta}=\left(\begin{array}{cccccccc}
0 & 0 & 0 & 0 & 0 & 0 & 0 & 0 \\
0 & 0 & 0 & 0 & 0 & 0 & 0 & 0 \\
0 & -2 \mu \frac{\partial}{\partial z} & 0 & 0 & 0 & 0 & 0 & 0 \\
-2 \mu \frac{\partial}{\partial x} & 0 & 0 & 0 & 0 & 0 & 0 & 0 \\
\mu \frac{\partial}{\partial z} & \mu \frac{\partial}{\partial x} & 0 & 0 & 0 & 0 & 0 & 0 \\
0 & -2 \mu E \frac{\partial}{\partial z} & 0 & 0 & 0 & 0 & 0 & 0 \\
-2 \mu E \frac{\partial}{\partial x} & 0 & 0 & 0 & 0 & 0 & 0 & 0 \\
\mu E \frac{\partial}{\partial z} & \mu E \frac{\partial}{\partial x} & 0 & 0 & 0 & 0 & 0 & 0
\end{array}\right)
$$


and using equations A.4 and A.11, we can write the gradient as

$$
\begin{aligned}
& \frac{\partial \epsilon}{\partial \eta}=-\sum_{s}<\frac{\partial \mathbf{A}}{\partial \eta} \mathbf{w}_{f}\left(\mathbf{x}, t ; \mathbf{x}_{s}\right), \mathbf{w}^{*}\left(\mathbf{x}, t ; \mathbf{x}_{s}\right)> \\
& =-\sum_{s}<\left(\begin{array}{cccccccc}
0 & 0 & 0 & 0 & 0 & 0 & 0 & 0 \\
0 & 0 & 0 & 0 & 0 & 0 & 0 & 0 \\
0 & -2 \mu \frac{\partial}{\partial z} & 0 & 0 & 0 & 0 & 0 & 0 \\
-2 \mu \frac{\partial}{\partial x} & 0 & 0 & 0 & 0 & 0 & 0 & 0 \\
\mu \frac{\partial}{\partial z} & \mu \frac{\partial}{\partial x} & 0 & 0 & 0 & 0 & 0 & 0 \\
0 & -2 \mu E \frac{\partial}{\partial z} & 0 & 0 & 0 & 0 & 0 & 0 \\
-2 \mu E \frac{\partial}{\partial x} & 0 & 0 & 0 & 0 & 0 & 0 & 0 \\
\mu E \frac{\partial}{\partial z} & \mu E \frac{\partial}{\partial x} & 0 & 0 & 0 & 0 & 0 & 0
\end{array}\right)\left(\begin{array}{c}
u \\
w \\
\sigma_{x x} \\
\sigma_{z z} \\
\sigma_{x z} \\
r_{x x} \\
r_{z z} \\
r_{x z}
\end{array}\right),\left(\begin{array}{c}
\hat{u} \\
\hat{w} \\
\hat{\sigma}_{x x} \\
\hat{\sigma}_{z z} \\
\hat{\sigma}_{x z} \\
\hat{r}_{x x} \\
\hat{r}_{z z} \\
\hat{r}_{x z}
\end{array}\right)> \\
& =-\sum_{s} \int\left(2 \mu \frac{\partial w}{\partial z} \hat{\sigma}_{x x}+2 \mu \frac{\partial u}{\partial x} \hat{\sigma}_{z z}-\left(\mu \frac{\partial u}{\partial z}+\mu \frac{\partial w}{\partial x}\right) \hat{\sigma}_{x z}-2 \mu E \frac{\partial w}{\partial z} \hat{r}_{x x}-2 \mu E \frac{\partial u}{\partial x} \hat{r}_{z z}+\mu E\left(\frac{\partial w}{\partial x}+\frac{\partial u}{\partial z}\right) \hat{r}_{x z}\right) d t .
\end{aligned}
$$

\section{REFERENCES}

Anderson, D. L., A. Ben-Menahem, and C. B. Archambeau, 1965, Attenuation of seismic energy in the upper mantle: Journal of Geophysical Research, 70, $1441-1448$.

Bai, J., and D. Yingst, 2013, Q estimation through waveform inversion: 75th Annual International Conference and Exhibition, EAGE, Extended Abstracts, Th-10-01.

Bai, J., D. Yingst, R. Bloor, and J. Leveille, 2014, Viscoacoustic waveform inversion of velocity structures in the time domain: Geophysics, 79(3), R103-R119. Blanch, J. O., J. O. Robertsson, and W. W. Symes, 1995, Modeling of a constant Q: Methodology and algorithm for an efficient and optimally inexpensive viscoelastic technique: Geophysics, 60(1), 176-184.

Boiero, D., M. Werning, and P. Vermeer, 2013, Q estimation from surface waves: 75th EAGE Conference \& Exhibition, 1-5.

Carcione, J. M., 2001, Wave fields in real media: Wave propagation in anisotropic, anelastic and porous media: Elsevier Science Ltd.

Dasgupta, R., and R. A. Clark, 1998, Estimation of Q from surface seismic reflection data: Geophysics, 63, 212-2128.

De Meersman, K., 2013, S-waves and the near surface: A time-lapse study of S-wave velocity and attenuation in the weathering layer of an alberta heavy oil field: The Leading Edge, 32, 40-47.

Dutta, G., and G. T. Schuster, 2016, Wave-equation Q-tomography: Geophysics, 81, 471-484.

Emmerich, H., and M. Korn, 1987, Incorporation of attenuation into time-domain computations of seismic wavefields: Geophysics, 52, $1252-1264$.

Gamar, F., D. Carotti, P. Guillaume, A. Gacha, and L. Lopes., 2015, Success of high-resolution volumetric Q-tomography in the automatic detection of gas anomalies on offshore brunei data: 2015 SEG Annual Meeting Abstract, 5184-5189.

Groos, L., M. Schafer, T. Forbriger, and T. Bohlen, 2014, The role of attenuation in 2D full-waveform inversion of shallow-seismic body and Rayleigh waves: Geophysics, 79, R24-R261. 
Hanafy, M. S., A. Altheyab, G. T. Schuster, 2015, Controlled noise seismology: 2015 SEG Annual Meeting Abstract, 5102-5107.

He, Y., J. Gao, and Z. Chen, 2015, On the comparison of properties of Rayleigh waves in elastic and viscoelastic media: International Journal of Numerical Analysis \& Modeling, 12, 254-267.

Ivanov, J., G. Tsoflias, R. D. Miller, S. L. Peterie, et al., 2014, Near-surface Qs and Qp estimations from Rayleigh waves using multi-channel analysis of surface waves (MASW) at an arctic ice-sheet site: 2014 SEG Annual Meeting Abstract, 2006-2011.

Jeng, Y., J. Y. Tsai, and S. H. Chen, 1999, An improved method of determining near-surface Q: Geophysics, 64, 1608-1617.

Kamei, R., and R. G. Pratt, 2008, Waveform tomography strategies for imaging attenuation structure with cross-hole data: 70th Annual International Conference and Exhibition, EAGE, Extended Abstracts, incorporating SPEEUROPEC, F019.

Komatitsch, D., Z. N. Xie, E. Bozdag, E. S. de Andrade, D. Peter, Q. Y. Liu, and J. Tromp, 2016, Anelastic sensitivity kernels with parsimonious storage for adjoint tomography and full waveform inversion: Geophysical Journal International, 204, 1-23.

Levander, A. R., 1988, Fourth-order finite-difference P-SV seismograms: Geophysics, 53, 1425-1436.

Li, J., and G. T. Schuster, 2016, Skeletonized wave equation of surface wave dispersion inversion: 2016 SEG Annual Meeting Abstract, 3630-3635.

Li, J., and S. M. Hanafy, 2016, Skeletonized inversion of surface wave: active source versus controlled noise comparison: Interpretation, 4, SH11-SH19.

Li, G., M. D. Sacchi, and H. Zheng, 2016, In situ evidence for frequency dependence of near-surface Q: Geophysical Journal International, 204, $1308-1315$.

Li, J., Z. C. Feng, and G. T. Schuster, 2017, Wave-equation dispersion inversion: Geophysical Journal International, doi: 10.1093/gji/ggw465.

Liao, O., and G. A. McMechan, 1995, 2.5D full-wavefield viscoacoustic inversion: Geophysical Prospecting, 43, 1043-1059

Lin, F. C., M. P. Moschetti, and M. H. Ritzwoller, 2008, Surface wave tomography of the western united states from ambient seismic noise: Rayleigh and Love wave phase velocity maps: Geophysical Journal International, 173, 281-298.

Luo, Y., and G. T. Schuster, 1991a, Wave-equation inversion of skeletalized geophysical data: Geophysical Journal International, 105, $289-294$.

Luo, Y., and G. T. Schuster, 1991b, Wave-equation traveltime inversion: Geophysics, 56, 645-653.

Nocedal, J., and S. Wright, 1999, Numerical Optimization: Springer Verlag.

Park, C. B., R. D. Miller, J. Xia, 1998, Imaging dispersion curves of surface waves on multi-channel record: 1998 SEG Annual Meeting Expanded Abstracts, $1377-1380$

Parolai, S., D. Bindi, A. Ansal, A. Kurtulus, A. Strollo, and J. Zschau, 2010, Determination of shallow S-wave attenuation by down-hole waveform deconvolution: a case study in Istanbul (Turkey): Geophysical Journal International, 181, 1147-1158.

Plessix, R. E., 2013, A review of the adjoint-state method for computing the gradient of a functional with geophysical applications: Geophysical Journal International, 167, 495-503.

Pinson, L. J., T. J. Henstock, J. K. Dix, and J. M. Bull, 2008, Estimating quality factor and mean grain size of sediments from high-resolution marine seismic data: Geophysics, 73, G19-G28.

Plessix, R., 2006, A review of the adjoint-state method for computing the gradient of a functional with geophysical applications: Geophysical Journal International, 167, 495-503.

Quan, Y. L., and J. M. Harris, 1997, Seismic attenuation tomography using the frequency shift method: Geophysics, 62, 895-905.

Robertsson, J. O., 1996, A numerical free-surface condition for elastic/viscoelastic finite-difference modeling in the presence of topography: Geophysics, 61,1921-1934. 
Robertsson, J. O., J. O. Blanch, and W. W. Symes, 1994, Viscoelastic finite-difference modeling: Geophysics, 59,1444-1456.

Schuster, G. T., 2017, Seismic Inversion: SEG Publishing: Tulsa, Oklahoma, doi.org/10.1190/1.9781560803423.

Strobbia, C., A. Laake, P. Vermeer, and A. Glushchenko, 2011, Surface waves: use them then lose them. surface-wave analysis, inversion and attenuation in land reflection seismic surveying: Near Surface Geophysics, 9, 503-514.

Wang, Y., and J. Zhang, 2014, Pseudo 2D elastic waveform inversion for Q factor in the near surface: 2014 SEG Annual Meeting Abstract, $2019-2023$.

Virieux, J., and S. Operto, 2009, An overview of full-waveform inversion in exploration geophysics: Geophysics, 74(6), WCC1-WCC26

Xia, J., R. D. Miller, and C. B. Park, 1999, Estimation of near-surface shear-wave velocity by inversion of Rayleigh wave: Geophysics, 64, 691-700.

Xia, J., R. D. Miller, C. B. Park, B. Choon and G. Tian, 2002, Determining Q of near-surface materials from Rayleigh waves: Journal of applied geophysics, 51, 121-129.

Xia, J., 2014, Estimation of near-surface shear-wave velocities and quality factors using multichannel analysis of surface-wave methods: Journal of applied geophysics, 103, 140-151.

Zhang, L., D. Zhu, and X. Zhang, 2015, Seismic attributes method for prediction of unconsolidated sand reservoirs of heavy oil: The Open Fuels \& Energy Science Journal, 8, 1-10.

Zhou, C., W. Cai, Y. Luo, G. T. Schuster, and S. Hassanzadeh, 1995, Acoustic wave-equation traveltime and waveform inversion of crosshole seismic data: Geophysics, 60, 765-773.

Zhu, T., J. M. Carcione, and J. M. Harris, 2013, Approximating constant-Q seismic propagation in the time domain: Geophysical Prospecting, 61, 931-940.

Zhu, T., and J. M. Harris, 2015, Improved estimation of P-wave velocity, S-wave velocity, and attenuation factor by iterative structural joint inversion of crosswell seismic data: Journal of Applied Geophysics, 123, 71-80.

This paper has been produced using the Blackwell Scientific Publications GJI LTEX2e class file. 